\title{
Progress and prospects for heavy flavour physics on the lattice
}

\author{
Carlos Pena* \\ Department of Theoretical Physics and IFT UAM-CSIC \\ Universidad Autónoma de Madrid \\ E-28049 Madrid, Spain \\ E-mail: carlos.pena@uam.es
}

I review recent progress in lattice computations relevant for $B$ - and charm physics, focusing on decay and mixing amplitudes with a direct impact on CKM analysis. Emphasis is put on the interplay with the upcoming new generation of experimental results, and the subsequent challenges for lattice computations in the heavy quark sector.

The 33rd International Symposium on Lattice Field Theory 14 -18 July 2015

Kobe International Conference Center, Kobe, Japan

* Speaker. 


\section{Introduction}

Flavour physics has long been one of the most powerful tools in the search for new physics, having led to key milestones in the construction of the Standard Model (SM). Nowadays, charm and bottom physics at the intensity frontier are pivotal to efforts to probe the limits of the Standard Model (SM).

Lattice QCD (LQCD) is the only known first-principles approach to the computation of longdistance strong-interaction contributions to flavour-changing processes in the quark sector. The maturity reached by simulation techniques in the last decade, which has resulted in a dramatic improvement of LQCD predictions for light quark physics, has more recently had a similar impact on the heavy quark sector: as will be discussed below, many decay and mixing processes that play a key role in the determination of Cabibbo-Kobayashi-Maskawa (CKM) matrix elements, as well as in unitarity triangle analysis of CKM consistency, can be now determined to very good precision. This in turn allows to fully exploit the large amount of experimental information produced by the BaBar and Belle B-factories. Yet, the new generation of results produced by such experiments as LHCb or BESIII, and, above all, the dramatic improvement in precision expected from the Belle II experiment [1], will pose a significant challenge to LQCD computations in the near future.

This review will focus on the computation of SM weak decay and mixing amplitudes of heavy mesons. A number of important topics (such as heavy quark masses, which require the discussion of a wholly different theoretical toolset, or amplitudes relevant for new physics models) will be sacrificed to brevity. New results for leptonic decays and $B^{0}-\bar{B}^{0}$ mixing will be summarised rapidly; more emphasis will be made on semileptonic decays, for which the last two years have witnessed a comparatively much more significant progress. The discussion will be mostly structured around the update of the Flavour Lattice Averaging Group (FLAG) review [2], the third edition of which is now finalised [3]. Detailed information about the new results presented at the conference can be found in the relevant proceedings contributions $[4,5,6,7,8]$.

\section{Reach of and formalisms for heavy quark physics on the lattice}

\subsection{Heavy quark scales in lattice simulations}

Any LQCD simulation involves an ultraviolet cutoff, given by the (inverse of the) lattice spacing $a$, and an infrared cutoff, given by the inverse of the spatial box size $L$. In order to bring cutoff effects under control, and allow for well-controlled continuum and infinite-volume limit extrapolations, all physical mass scales $M$ in the problem addressed need to be far away from the cutoffs, $L^{-1} \ll M \ll a^{-1}$. Apart from the intrinsic strong interaction scale $\Lambda_{\mathrm{QCD}}$, this applies to the values of hadron masses for all the flavours involved in the computation.

Infrared cutoff effects most often take the form of finite-volume corrections to correlation functions, which for large enough volumes $\left(m_{\pi} L \gtrsim 4\right.$ being a reasonable rule of thumb) behave $\sim e^{-m_{\pi} L}$; this implies that computations at the physical values of light quark masses must take place in boxes of size $L \gtrsim 6 \mathrm{fm}$, and pions twice heavier than their physical mass still require $L \gtrsim 3 \mathrm{fm}$. Ultraviolet cutoff effects, on the other hand, are generally expected to be power-like, based on Symanzik effective theory. Assuming that all $\mathrm{O}(a)$ effects are removed, either by the properties of the regularisation, or by the inclusion of appropriate counterterms to the lattice action and composite operators, the leading cutoff effects driven by quark masses will be $\sim\left(a m_{q}\right)^{2}$. This means that lattice spacings $a \lesssim 0.1 \mathrm{fm}$ are (naively) enough to keep effects related to the light $u, d, s$ quarks at the few percent level, but significantly smaller lattice spacings are needed for charm- and $B$-physics: for instance, $\left(a m_{q}\right)^{2} \lesssim 0.2$ implies $a \lesssim 0.07 \mathrm{fm}$ for $q=c$, and $a \lesssim 0.02 \mathrm{fm}$ for $q=b$. Thus, in this naive counting, lattice sizes $(L / a) \gtrsim 90$ and $(L / a) \gtrsim 300$, respectively, would be needed to keep scaling violations at the $20 \%$ ballpark. ${ }^{1}$

\footnotetext{
${ }^{1}$ Evidently, smaller lattices can be used at the price of introducing systematic uncertainties coming from chiral extrapolations from heavy pion masses.
} 

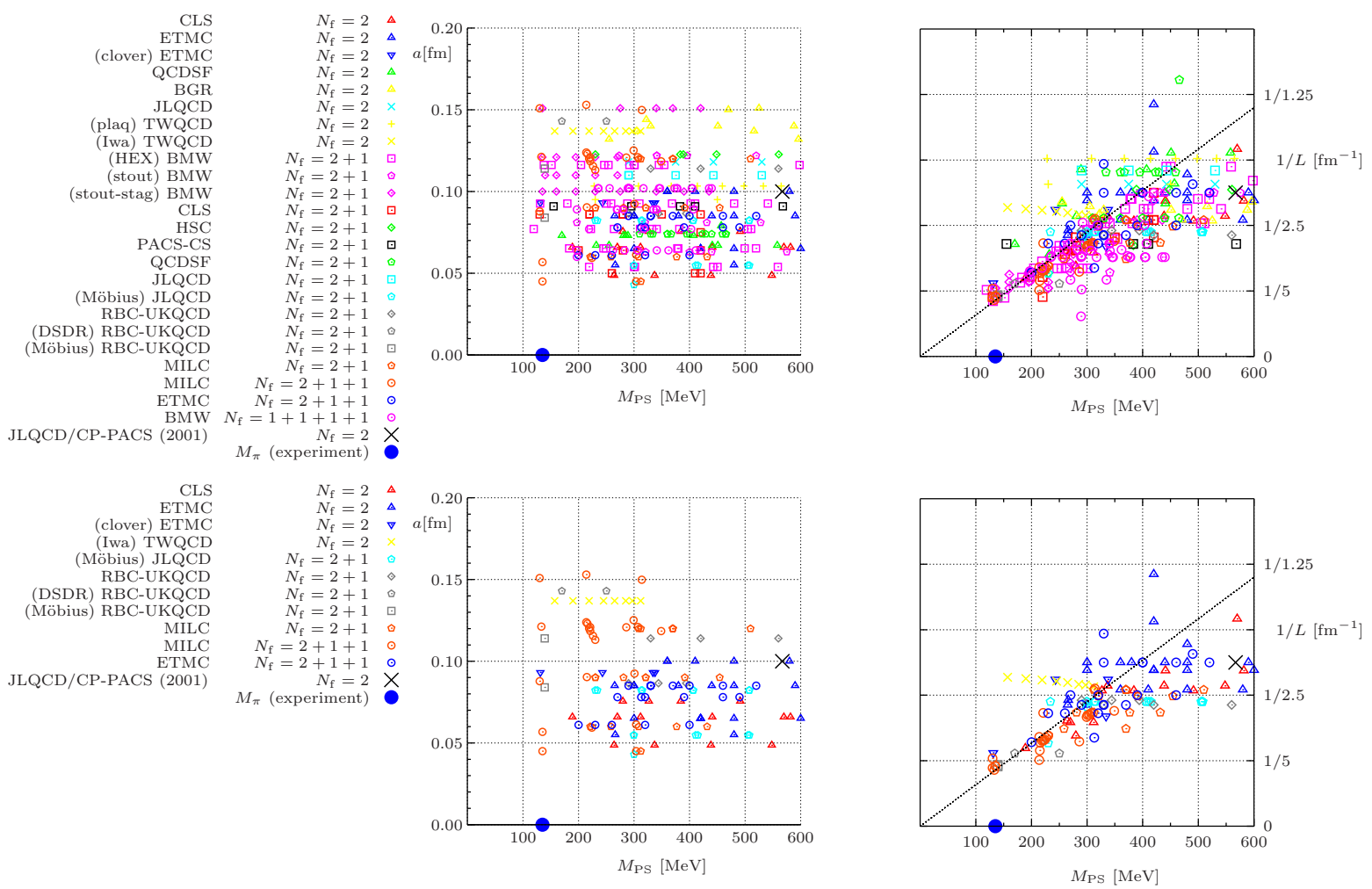

Figure 1: Top: LQCD simulation landscape as of Summer 2015. Ensembles are shown in the plane spanned by the sea pion mass $M_{\mathrm{PS}}$ and the lattice spacing $a$ (left), and in the plane spanned by $M_{\mathrm{PS}}$ and the spatial box size $L$ (right; the straight line corresponds to $M_{\mathrm{PS}} L=4$ ). Bottom: same as above, now showing only the ensembles used in computations that will be discussed in this review. Figures courtesy of G. Herdoíza.

Current state-of-the-art simulations are summarised in Fig. 1. The size of the region covered by simulations in the $\left(a, m_{\pi}, L\right)$ parameter space is determined by the largest computational cost attainable with current supercomputing resources, which in turn depends on algorithm efficiency and Moore's law. One immediate conclusion is that, while charm physics is affordable (though still at the price of working with relatively large pion masses), direct simulation of $b$ quarks is still very difficult — indeed, within the accessible region $\left(a m_{b}\right)^{2} \gtrsim 1$. As a consequence, the study of $B$-physics on the lattice requires input from effective theory, that exploits in various ways the large value of the $b$-quark mass - or, more precisely, the small value of the ratio $\Lambda_{\mathrm{QCD}} / m_{b}$ - to bypass or assist a direct simulation. The resulting different methodologies will be briefly summarised below. Another point worth stressing is that only a fraction of the available ensembles have been used so far for heavy quark physics. This is partly due to the fact that the coarser ensembles lead to larger cutoff effects, and partly due to the need of fully assessing light quark physics before proceeding to the heavy sector.

Prospects for direct simulations of $b$ quarks at their physical mass crucially depend on the scaling behaviour of algorithmic cost with decreasing $a$. Until recently, typical cost estimates included a powerlaw behaviour in $a$, cf. e.g. the $a^{-6}$ scaling law quoted in [9]. It has now been recognised, however, that the performance of common algorithms used in lattice simulations deteriorates very rapidly for values of the lattice spacing $a \lesssim 0.05 \mathrm{fm}$, leading to a surge in autocorrelation times - especially for quantities very sensitive to long-distance physics - which is furthermore essentially insensitive to the values of sea quark masses [10]. One particular consequence is the inability of the algorithm to change topological sector, which has led to the moniker "topology freezing" for this behaviour. ${ }^{2}$ Another

\footnotetext{
${ }^{2}$ Initial evidence mostly relied on simulations of the pure gauge theory, and of $N_{\mathrm{f}}=2$ QCD with Wilson fermions. Similar findings have recently been reported in $N_{\mathrm{f}}=2+1$ and $N_{\mathrm{f}}=2+1+1$ simulations with rooted staggered fermions at lattice spacings below $0.05 \mathrm{fm}[11,5]$.
} 
obvious consequence is the impossibility to access directly the $b$-quark mass region within standard simulation setups.

The existing proposals to avoid the algorithmic critical slowing down for $a \lesssim 0.05$ fm involve abandoning the (anti)periodic boundary conditions used in most QCD simulations. For instance, the introduction of open boundary conditions in Euclidean time - i.e. the substitution of the periodic torus for an open-ended cylinder - has been shown to improve the scaling of the algorithm considerably [12], and has been incorporated into the latest generation of large-scale simulations by the Coordinated Lattice Simulations effort [13]. The rationale for this approach is that open boundary conditions allow topological structures to flow in and out of the lattice. Another very recent proposal [14] replaces the periodic torus by a non-orientable manifold, leading to so-called "P-periodic" boundary conditions in Euclidean time. This results in a similar scaling law for autocorrelation times as open boundary conditions, albeit autocorrelation times remain significantly larger than in the latter approach. On the other hand, open boundary conditions break translation invariance in the time direction and give rise to significant boundary effects, both of which are argued to be absent with P-periodic boundary conditions. Finally, in [15] a multiscale algorithm is applied to the pure Yang-Mills theory in a periodic lattice, again showing significant promise in the reduction of autocorrelations. While it is still unclear which is the true potential of these new methodologies in terms of reaching the $a \sim 0.01 \mathrm{fm}$ region, their availability is a crucial step towards a significant improvement of our control on the systematics of lattice $B$-physics computations.

\subsection{Approaches to heavy quark physics}

As discussed above, the unavailability of LQCD simulations at lattice spacings below $a \approx 0.05 \mathrm{fm}$ poses a huge challenge for $B$-physics computations, since using a similar setup as for light quarks will result in extremely large cutoff effects. Existing approaches to $B$-physics thus rely on input from effective descriptions of the heavy quark dynamics. In broad terms, this implies that an expansion in powers of $\Lambda_{\mathrm{QCD}} / m_{\mathrm{h}}$ (where $m_{\mathrm{h}}$ is the mass of the heavy quark) underlies the procedure, and that some assumptions are made at the field-theoretical level, including the size of corrections neglected by the truncation of the expansion.

There are two main procedures to perform the expansion: Heavy Quark Effective Theory (HQET) [16], and Non-Relativistic QCD (NRQCD) [17]. HQET provides the correct asymptotic description of QCD correlation functions in the static limit $\left|\mathbf{p}_{\mathrm{h}}\right| / m_{\mathrm{h}} \rightarrow 0$. Subleading effects are described by higher dimensional operators, whose coupling constants are formally of $O\left(\left(1 / m_{\mathrm{h}}\right)^{n}\right)$. The HQET expansion works well for heavy-light systems in which the heavy-quark momentum is small compared to the mass. In the static limit the $b$ quark is described by a theory of a static fermion field coupled to the gauge field, and $b$ propagators are replaced by Wilson lines in QCD correlation functions; the resulting theory is renormalisable, and computations can be carried out efficiently. NRQCD, on the other hand, is constructed by matching the effective theory to full QCD order by order in the heavy-quark velocity $v_{b}^{2}$ (for heavy-heavy systems) or in $\Lambda_{\mathrm{QCD}} / m_{\mathrm{h}}$ (for heavy-light systems), and in powers of $\alpha_{\mathrm{s}}$. Relativistic corrections appear as higher-dimensional operators in the Hamiltonian. As an effective field theory, NRQCD is only useful with an ultraviolet cutoff of order $m_{\mathrm{h}}$ or less.

The use of effective theory implies that all approaches suffer from systematic uncertainties, although the extent to which they can be assessed differs widely. In any case, it is crucial to cross-check the results from different procedures in order to gain confidence about systematic error estimates. Below we summarise the main features of each family of currently-used approaches. A cartoon for each of them is provided in Fig. 2. ${ }^{3}$

\subsubsection{Nonperturbative HQET}

A framework to treat HQET nonperturbatively was introduced in [18]. The rationale for this approach

\footnotetext{
${ }^{3}$ In general, lattice collaborations stick to one of the methods, as mentioned in the discussion below. Thus, in the coming sections the formalism employed for $b$ quarks in any given calculation will often not be indicated explicitly.
} 


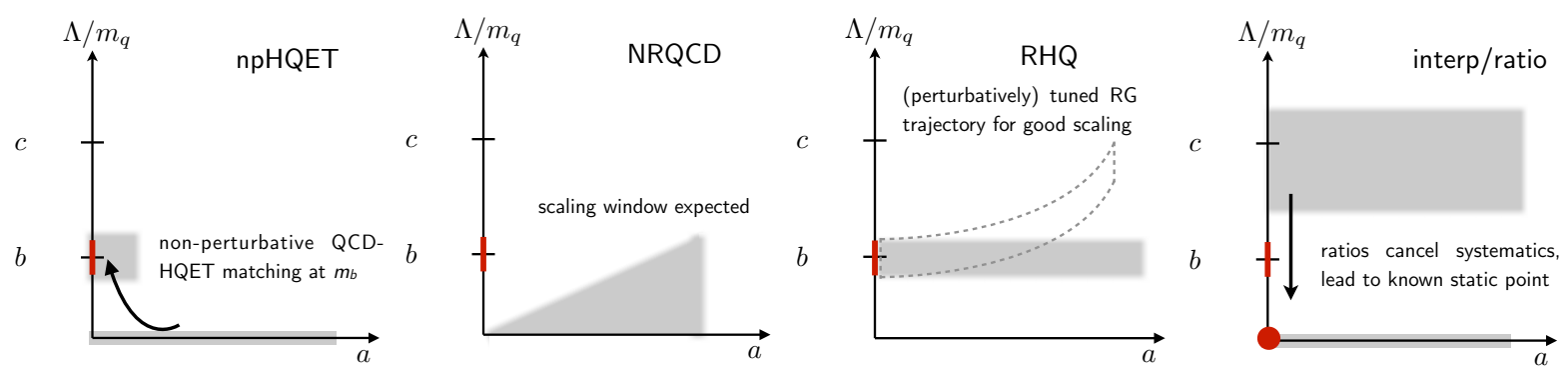

Figure 2: Cartoon depicting various approaches to heavy quark physics on the lattice in the plane spanned by the lattice spacing $a$ and the inverse heavy quark mass.

is based on the observation [19] that, while $\alpha_{\mathrm{s}}\left(m_{\mathrm{h}}\right)$ decreases logarithmically with $m_{\mathrm{h}}$, corrections in the effective theory are power-like in $\Lambda_{\mathrm{QCD}} / m_{\mathrm{h}}$; therefore, it is possible that the leading errors in a calculation will be due to the perturbative matching of the action and the currents at a given order $\Lambda_{\mathrm{QCD}} / m_{\mathrm{h}}$, rather than to the truncation of the heavy-quark expansion. To eliminate that systematics, the order of the expansion is fixed, and the matching to QCD is performed nonperturbatively beyond leading order in $\Lambda_{\mathrm{QCD}} / m_{\mathrm{h}}$. Higher-dimensional interaction terms in the effective Lagrangian are treated as spacetime volume insertions into static correlation functions, thus guaranteeing that, at any order in the heavy-quark expansion, the effective theory is renormalisable.

The implementation used by the ALPHA Collaboration employs two steps, involving separate sets of simulations. First, the couplings of the effective theory are determined by matching QCD and HQET nonperturbatively in small physical volumes, where values of the lattice spacing that allow for a full relativistic treatment of the $b$ quark are reachable. Then HQET is simulated in large volumes to compute hadronic observables. In order to avoid relevant systematics related to the matching in finite volume, the corresponding length scale $L$ is chosen such that higher orders in $\left(m_{\mathrm{h}} L\right)^{-1}$ and $\Lambda_{\mathrm{QCD}} / m_{\mathrm{h}}$ are of comparable size.

\subsubsection{NRQCD}

In a lattice implementation [20], the NRQCD requirement to work with an ultraviolet cutoff not above $m_{\mathrm{h}}$ translates into $a m_{\mathrm{h}} \gtrsim 1$, which implies that $a$ has to be kept above a minimum value at fixed heavy quark mass, and a continuum limit cannot be taken. Lattice NRQCD results thus unavoidably retain a cutoff dependence, though they are expected to be fairly independent of the cutoff within some scaling window, where physics can be extracted. One advantage of NRQCD is its ability to tackle heavy-light and heavy-heavy systems using the same action.

In order to optimise the approach, practical implementations (in particular, the one employed by the HPQCD Collaboration) include counterterms in the action that subtract the largest cutoff effects perturbatively to some fixed order in a simultaneous expansion in powers of $\alpha_{\mathrm{s}}$ and $\Lambda_{\mathrm{QCD}} / m_{\mathrm{h}}-$ see e.g. [21] for details. For almost all of the explored quantities, this usually leads to error budgets where the largest contribution comes from the $\mathscr{O}\left(\alpha_{\mathrm{s}}^{2}\right)$ uncertainty in the perturbative matching of operators to full QCD.

\subsubsection{Relativistic heavy-quark actions}

Relativistic heavy-quark (RHQ) actions are designed to remove large $\mathscr{O}\left(\left(a m_{\mathrm{h}}\right)^{n},\left(a\left|\mathbf{p}_{\mathrm{h}}\right|\right)^{n}\right)$ cutoff effects by adjusting the coefficients of suitable higher-dimensional counterterms via a Symanzik-like procedure. At fixed lattice spacing, RHQ formulations are expected to smoothly interpolate between the light-quark and static limits, which are recovered when $a m_{\mathrm{h}} \ll 1$ and $a m_{\mathrm{h}} \gg 1$, respectively. A general framework for the approach was developed in [22].

The three most widely used implementations of this idea are the so-called Fermilab interpretation, commonly employed by FNAL/MILC [22]; the RHQ "Columbia" formulation developed by Li, Lin and Christ, in current use by RBC/UKQCD [23]; and the Tsukuba heavy-quark action introduced in [24]. 
All employ an anisotropic action with a Sheikholeslami-Wohlert term [25], and use HQET to constrain the mass dependence of the action and composite operator improvement coefficients in order to attain the desired scaling properties. The main difference between Fermilab and Columbia is that, while in the former case perturbation theory is used to determine the coefficients, thus leading to truncation errors of $\mathscr{O}\left(\alpha_{\mathrm{s}} a\left|\mathbf{p}_{\mathrm{h}}\right|,\left(a\left|\mathbf{p}_{\mathrm{h}}\right|\right)^{2}\right)$ at the action level, in the Columbia action the coefficients are tuned nonperturbatively by reproducing a set of spectral observables at finite lattice spacing. The Tsukuba action, on the other hand, allows for further anisotropies in both dimension- 4 and dimension- 5 operators in the action, and uses a nonperturbative determination of the Sheikholeslami-Wohlert coefficient in the massless limit together with perturbation theory to track the mass dependence of action coefficients.

\subsubsection{Interpolation procedures}

In this approach static limit results are combined with full QCD computations, performed at the largest directly accessible values of the heavy quark mass (i.e. at or slightly above the charm scale). This allows for an interpolation in $\Lambda_{\mathrm{QCD}} / m_{\mathrm{h}}$, that provides the value of the observable of interest at $m_{\mathrm{h}}=m_{b}$. Such an interpolation can be guided by using HQET predictions for the heavy quark mass dependence.

A number of specific variants of this general idea have been proposed [26]. One implementation widely used in recent computations by the ETM Collaboration and some related efforts, dubbed "ratio method" [27], involves considering ratios of values of the observable of interest computed at different values of the heavy quark mass, keeping the ratio between consecutive mass values constant. Ratios are built such that their static limit is trivial. This has a double advantage: uncertainties from the static value are eliminated, and a number of systematic uncertainties are either absent or largely cancelled in the ratios — in particular the bulk of the large truncation effects [28] induced by a perturbative matching between QCD and HQET.

\section{Leptonic decays}

The SM branching fraction for the charged-current-mediated decay of a $D_{(s)}$ meson is given by

$$
\frac{\mathscr{B}\left(D_{(s)} \rightarrow \ell v_{\ell}\right)}{\tau_{D_{(s)}}}=\frac{G_{\mathrm{F}}^{2}\left|V_{c q}\right|^{2}}{8 \pi} f_{D_{(s)}}^{2} m_{\ell}^{2} m_{D_{(s)}}\left(1-\frac{m_{\ell}^{2}}{m_{D_{(s)}}^{2}}\right)^{2}
$$

with $q=d, s$, and where the long-distance QCD contribution is encoded in the decay constant $f_{D_{(s)}}=$ $\left\langle 0\left|\bar{c} \gamma^{\mu} \gamma_{5} q\right| D_{(s)}\right\rangle /\left(i p_{D_{(s)}}^{\mu}\right)$. An experimental measurement of $\mathscr{B}$ can then be used to determine the CKM matrix element $\left|V_{c q}\right|$, with theory uncertainties (apart from the largely subdominant higher-order OPE terms) given by the error on $f_{D_{(s)}}$ and the neglect of electromagnetic corrections, and experimental uncertainties (other than the one on $\mathscr{B}$ itself) dominated by the error on the meson lifetime $\tau_{D_{(s)}}$. The latter two sources of error are $\sim \mathscr{O}(1 \%)$.

An up-to-date summary of experimental results for leptonic charm decay can be found e.g. in [29, 30]. The current precision on $\mathscr{B}\left(D_{s}^{+} \rightarrow \mu^{+} v_{\mu}\right)$ and $\mathscr{B}\left(D_{s}^{+} \rightarrow \tau^{+} v_{\tau}\right)$ quoted by the PDG is $4.5 \%$ and $4.3 \%$, respectively, while a recent preliminary measurement of $\mathscr{B}\left(D^{+} \rightarrow \mu^{+} v_{\mu}\right)$ by BESIII has significantly improved the precision of this channel, bringing it at $4.5 \%$ [31]. Meanwhile, prospects for Belle II point at a precision for $D_{s} \rightarrow \tau \nu_{\tau}$ in the interval 2.3\%-3.6\% with the long-term expected $50 \mathrm{ab}^{-1}$ of data, while for $D_{s} \rightarrow \mu v_{\mu}$ a precision around $1 \%$ is expected [32]. Note that at that level of precision electromagnetic corrections many no longer be negligible in this decay.

The most significant progress in lattice determinations of charm decay constants after FLAG-2 is the extremely precise $N_{\mathrm{f}}=2+1+1$ computation by FNAL/MILC using HISQ quarks [33], which, together with the final value by ETM [34], allows to quote averages with a precision better than $0.7 \%$ for $f_{D}$ and $0.5 \%$ for $f_{D_{s}}$; theoretical improvement on these figures thus requires a serious attack on electromagnetic corrections. A computation by $\chi \mathrm{QCD}[35]$ has meanwhile slightly improved the precision 

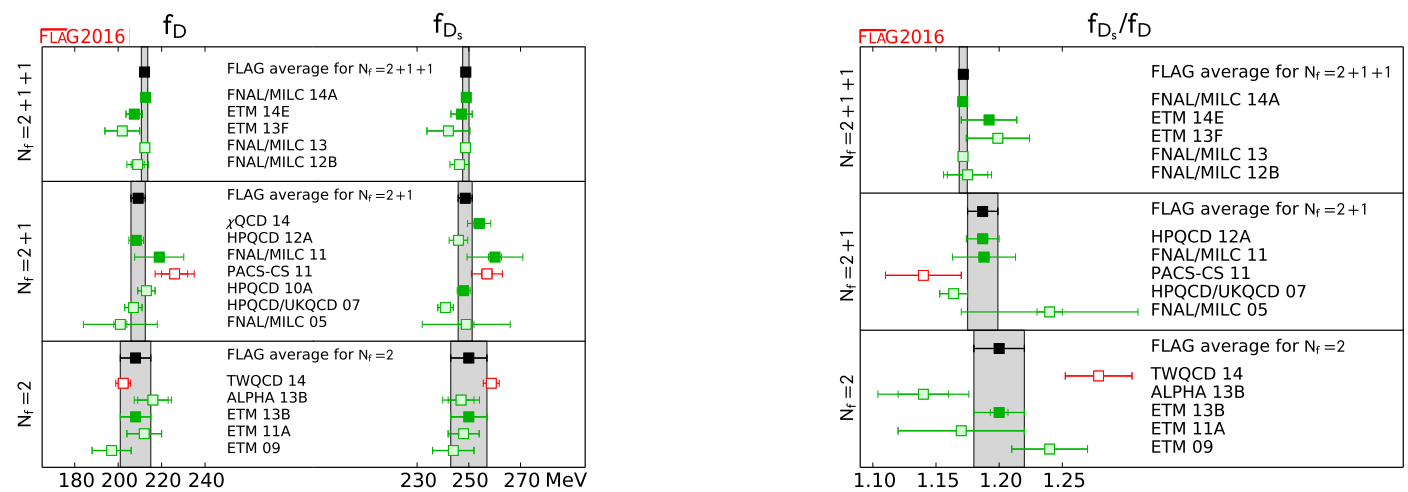

Figure 3: FLAG-3 summary plots for leptonic $D_{(s)}$ decay constants (see [3] for a complete list of references).
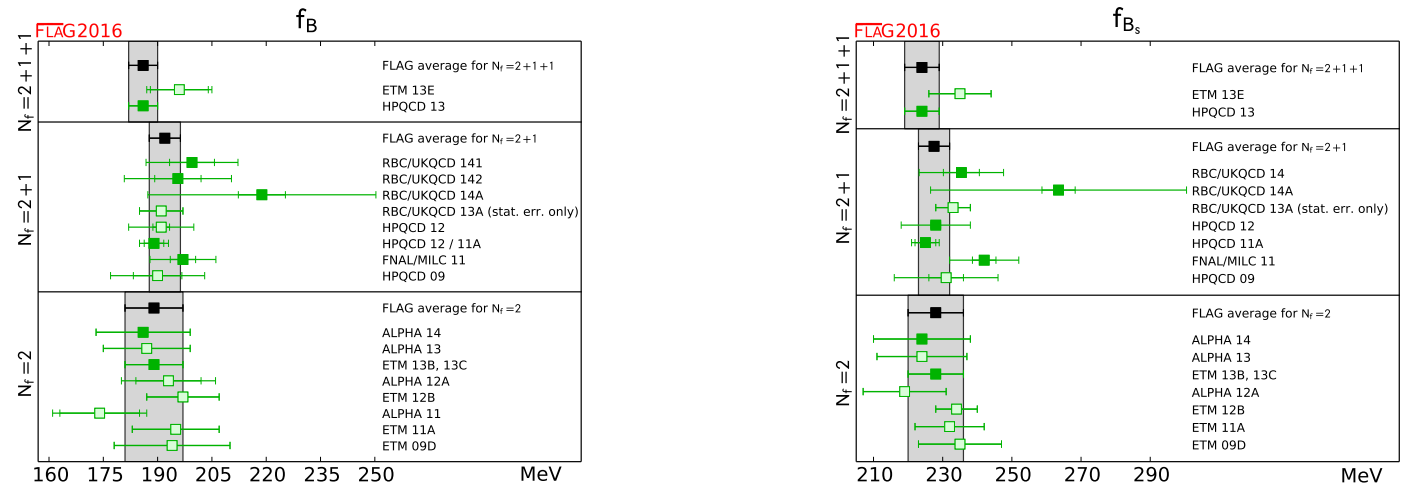

Figure 4: FLAG-3 summary plots for leptonic $B_{(s)}$ decay constants (see [3] for a complete list of references).

\begin{tabular}{l|lll|lll}
\hline \multicolumn{1}{c|}{$N_{\mathrm{f}}$} & \multicolumn{1}{c}{$f_{D}[\mathrm{MeV}]$} & \multicolumn{1}{c}{$f_{D_{s}}[\mathrm{MeV}]$} & \multicolumn{1}{c}{$f_{D_{s}} / f_{D}$} & $f_{B}[\mathrm{MeV}]$ & $f_{B_{s}}[\mathrm{MeV}]$ & \multicolumn{1}{c}{$f_{B_{s}} / f_{B}$} \\
\hline $2+1+1$ & $212.15(1.45)$ & $248.83(1.27)$ & $1.1716(32)$ & $186(4)$ & $224(5)$ & $1.205(7)$ \\
$2+1$ & $209.2(3.3)$ & $249.8(2.3)$ & $1.187(12)$ & $192.0(4.3)$ & $228.4(3.7)$ & $1.201(16)$ \\
2 & $208(7)$ & $250(7)$ & $1.20(2)$ & $188(7)$ & $227(7)$ & $1.206(23)$ \\
\hline
\end{tabular}

Table 1: FLAG-3 averages for leptonic $D_{(s)}$ and $B_{(s)}$ decay constants.

of the $N_{\mathrm{f}}=2+1$ determination. A new result for $N_{\mathrm{f}}=2$ with limited control on systematics has also been provided by TWQCD [36]. The new FLAG averages are given in Table 1 and Fig. 3.

The SM branching fraction for the leptonic decay of $B^{+}$and $B_{c}^{+}$mesons is given by Eq. (3.1), after replacing meson masses, decay constants, and CKM matrix elements appropriately. The branching fraction for leptonic $B_{s}^{0}$ decay, which proceeds at one loop in the SM electroweak interaction, is instead

$$
\frac{\mathscr{B}\left(B_{s} \rightarrow \ell v_{\ell}\right)}{\tau_{B_{s}}}=\frac{G_{\mathrm{F}}^{2}\left|V_{t b}^{*} V_{t s}\right|^{2}}{\pi} f_{B_{s}}^{2} Y\left(\frac{\alpha}{4 \pi \sin ^{2} \theta_{W}}\right)^{2} m_{B_{s}} m_{\ell}^{2} \sqrt{1-4 \frac{m_{\ell}^{2}}{m_{B_{s}}^{2}}},
$$

where $Y$ is a function that includes NLO QCD and electroweak corrections. All the decay constants involved are given by the meson-to-vacuum matrix element of an axial current $\bar{b} \gamma^{\mu} \gamma_{5} q ; q=u, s, c$.

While the tree-level $B^{+}$and $B_{c}^{+}$decays can provide determinations of $\left|V_{q b}\right|(q=u, c), B_{s}$ leptonic decay is a powerful probe of new physics. The current experimental value $\mathscr{B}\left(B_{s}^{0} \rightarrow \mu^{+} \mu^{-}\right)=$ $2.8_{-0.6}^{+0.7} \times 10^{-9}$ [37] is well-compatible with the SM prediction. In the case of tree-level decays, only the $B^{+} \rightarrow \tau^{+} v_{\tau}$ channel has been measured by both BaBar [38] and Belle [39], in both cases using different tagging methods. The uncertainties of these measurements are significantly large, with precisions of at best $40 \%$; furthermore, central values tend to be larger than expected from CKM fits and the more precise semileptonic channels (cf. below). Uncertainties on lifetime, higher-order OPE and 

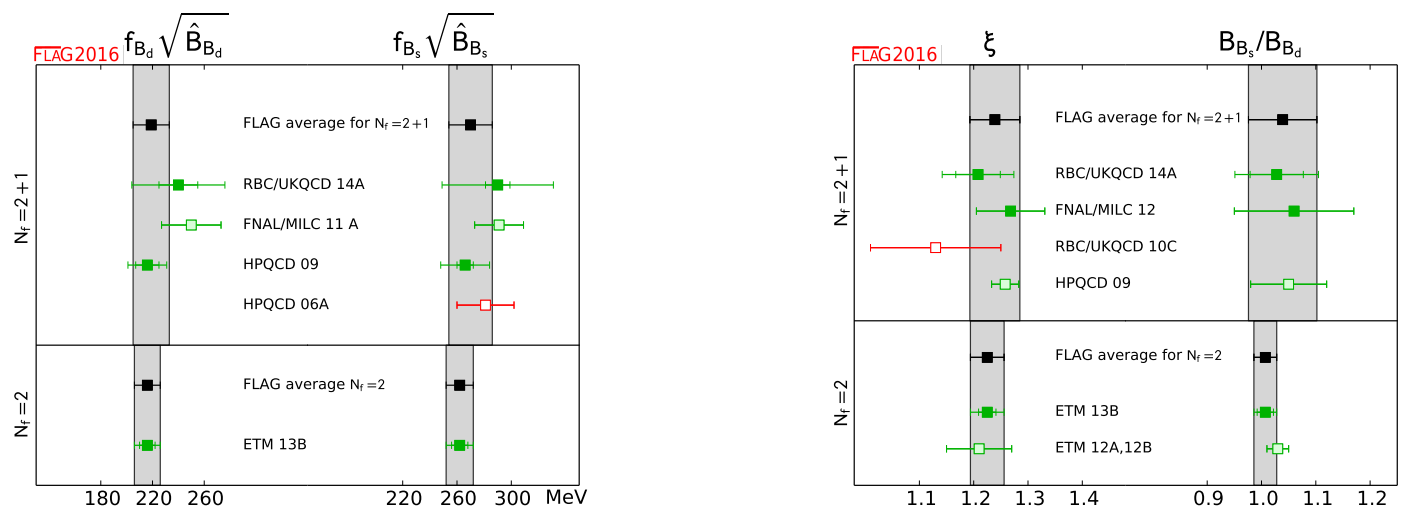

Figure 5: FLAG-3 summary plots for $B$-meson bag parameters (see [3] for a complete list of references).

\begin{tabular}{l|ll|cc|cc}
\hline$N_{\mathrm{f}}$ & $f_{B_{d}} \sqrt{\hat{B}_{B_{d}}}[\mathrm{MeV}]$ & $f_{B_{s}} \sqrt{\hat{B}_{B_{s}}}[\mathrm{MeV}]$ & $\hat{B}_{B_{d}}$ & $\hat{B}_{B_{s}}$ & $\xi$ & $\hat{B}_{B_{s}} / \hat{B}_{B_{d}}$ \\
\hline $2+1$ & $219(14)$ & $270(16)$ & $1.26(9)$ & $1.32(6)$ & $1.239(46)$ & $1.039(63)$ \\
2 & $216(10)$ & $262(10)$ & $1.30(6)$ & $1.32(5)$ & $1.225(31)$ & $1.007(21)$ \\
\hline
\end{tabular}

Table 2: FLAG-3 averages for $B$-meson bag parameters.

kinematic factors are very small; the dominant sources of theory uncertainty are the decay constants and missing electromagnetic corrections, and are however very small compared with the error on $\mathscr{B}$ itself. Thus, while this is an exciting channel in the search for new physics, better experimental precision is required to establish meaningful comparisons with other exclusive determinations of $\left|V_{u b}\right|$. Belle II projections [32] expect a dramatic improvement with a $5 \%$ precision for $\mathscr{B}\left(B^{+} \rightarrow \tau^{+} v_{\tau}\right)$ with the full $50 \mathrm{ab}^{-1}$ dataset, while a $5 \sigma$ measurement of $\mathscr{B}\left(B^{+} \rightarrow \mu^{+} v_{\mu}\right)$ is also foreseen. ${ }^{4}$

On the theory side, relatively little progress has been made after the FLAG-2 review concerning determinations of $f_{B}$ and $f_{B_{s}}$ : new results include the final npHQET $N_{\mathrm{f}}=2$ ALPHA values [41], new $N_{\mathrm{f}}=2+1$ results from RBC/UKQCD with relatively large errors [42, 43], using a RHQ formulation for the $b$ quark, and preliminary $N_{\mathrm{f}}=2+1+1$ results by ETM [44] using their ratio method. The current situation is summarised in Table 1 and Fig. 4. While the uncertainties are generally larger than for charm decay, due to the complications related to the treatment of $b$ quarks, the precision ballpark is already at the few percent level, thus ahead of the foreseeable experimental precision.

\section{4. $B^{0}-\bar{B}^{0}$ mixing}

Neutral $B$-meson mixing is induced in the SM, to lowest order in the electroweak theory, through oneloop box diagrams, resulting in an effective $\Delta B=2$ weak Hamiltonian of the form

$$
H_{\mathrm{w}}^{\Delta B=2}=\frac{G_{\mathrm{F}}^{2} M_{W}^{2}}{16 \pi^{2}} S_{0}\left(\frac{m_{t}^{2}}{M_{W}^{2}}\right) \eta_{2 B}\left[\left(V_{t d}^{*} V_{t b}\right)^{2} Q_{1}^{d}+\left(V_{t s}^{*} V_{t b}\right)^{2} Q_{1}^{s}\right], \quad Q_{1}^{q}=\left(\bar{b}_{\mathrm{L}} \gamma_{\mu} q_{\mathrm{L}}\right)\left(\bar{b}_{\mathrm{L}} \gamma_{\mu} q_{\mathrm{L}}\right),
$$

where electroweak and high-energy QCD corrections are contained in the Inami-Lim function $S_{0}$ and the factor $\eta_{2 B}$, respectively. $\hat{Q}_{1}^{q}$ indicates the renormalisation group invariant (RGI) operator, obtained from the renormalised operator at scale $\mu$ by calculating the anomalous dimension and beta function appearing in the renormalisation group equations for the operator and the strong coupling constant,

$$
\mu \frac{\partial}{\partial \mu} \bar{Q}(\mu)=\gamma(\bar{g}(\mu)) \bar{Q}(\mu), \quad \mu \frac{\partial}{\partial \mu} \bar{g}(\mu)=\beta(\bar{g}(\mu)),
$$

\footnotetext{
${ }^{4}$ The leptonic decay of the charmed $B_{c}^{+}$meson has not been measured experimentally yet, but a SM prediction exists based on the HPQCD computation of the relevant decay constant [40].
} 
with perturbative expansions $\gamma(g)=-\gamma_{0} g^{2}+\ldots$ and $\beta(g)=-b_{0} g^{3}+\ldots$, and computing

$$
\hat{Q}=\left[\frac{\bar{g}^{2}(\mu)}{4 \pi}\right]^{-\frac{\gamma_{0}}{2 b_{0}}} \exp \left\{-\int_{0}^{\bar{g}(\mu)} \mathrm{d} g\left[\frac{\gamma(g)}{\beta(g)}-\frac{\gamma_{0}}{b_{0} g}\right]\right\} \bar{Q}(\mu) .
$$

Long-distance QCD contributions are encoded in the bag parameters

$$
\hat{B}_{B_{q}}=\frac{\left\langle\bar{B}_{q}^{0}\left|\hat{Q}_{1}^{q}\right| B_{q}^{0}\right\rangle}{\frac{8}{3} f_{B_{q}}^{2} m_{B_{q}}^{2}}, \quad \xi^{2}=\frac{f_{B_{s}}^{2} \hat{B}_{B_{s}}}{f_{B_{d}}^{2} \hat{B}_{B_{d}}},
$$

where we have also defined the ratio $\xi$, that will be discussed below. Note that $\hat{B}_{B_{q}}$ and $\xi$ are scale- and renormalisation-scheme-independent by construction.

A non-zero mixing amplitude results in mass differences between the $\mathrm{CP}$ eigenstates of the neutral meson system, for which the SM prediction is

$$
\Delta m_{q}=\frac{G_{\mathrm{F}}^{2} M_{W}^{2} m_{B_{q}}}{6 \pi^{2}}\left|V_{t q}^{*} V_{t b}\right|^{2} S_{0}\left(\frac{m_{t}^{2}}{M_{W}^{2}}\right) \eta_{2 B} f_{B_{q}}^{2} \hat{B}_{B_{q}} .
$$

These quantities are experimentally measurable to high precision — current PDG averages [31] quote $0.6 \%$ and $0.1 \%$ for $\Delta m_{d}$ and $\Delta m_{s}$, respectively. Another interesting observable is the ratio $\Delta m_{s} / \Delta m_{d}$, where short-distance effects cancel and the long-distance QCD contribution is encoded in the ratio $\xi$ in Eq. (4.4), which can be computed on the lattice to significantly better precision than the individual $f_{B_{q}}^{2} \hat{B}_{B_{q}}$. Using a measurement of $\Delta m_{q}$ and a computation of $f_{B_{q}}^{2} \hat{B}_{B_{q}}$ it is then possible to determine $\left|V_{t q}^{*} V_{t b}\right|^{2}$, and feed it to a unitarity triangle analysis on the $\bar{\rho}-\bar{\eta}$ plane of Wolfenstein parameters, where, given the values of $\left|V_{t q}\right|$, it constrains the position of the triangle apex to lie on a circumference. Since the current precision on the knowledge of the relevant combination of CKM moduli is in the 7-8\% ballpark, this sets the precision target on $\hat{B}_{B_{q}}$ to avoid dominant theory uncertainties.

Unfortunately, relatively few results exist yet for $B$-meson bag parameters and/or their ratios; and the only update from the FLAG- 2 review is an $N_{\mathrm{f}}=2+1$ RBC/UKQCD computation using a RHQ treatment for the $b$ quark [43], with however significantly larger errors than the pre-existing HPQCD [45] and FNAL/MILC [46] results. Together with the now-published ETM values for $N_{\mathrm{f}}=2$ [34], this results in the landscape illustrated by Fig. 5 and Table 2.

\section{Semileptonic decays}

\section{1 $D$-meson decays}

The SM differential rate for $D \rightarrow P \ell v_{\ell}$ decay with $P=\pi, K(q=d, s)$ is given by

$$
\begin{aligned}
\frac{\mathrm{d} \Gamma\left(D \rightarrow P \ell v_{\ell}\right)}{\mathrm{d} q^{2}}=\frac{G_{\mathrm{F}}^{2}\left|V_{c q}\right|^{2}}{24 \pi^{3}} \frac{\left(q^{2}-m_{\ell}^{2}\right)^{2} \sqrt{E_{P}^{2}-m_{P}^{2}}}{q^{4} m_{D}^{2}}[ & \left(1+\frac{m_{\ell}^{2}}{2 q^{2}}\right) m_{D}^{2}\left(E_{P}^{2}-m_{P}^{2}\right)\left|f_{+}\left(q^{2}\right)\right|^{2} \\
& \left.+\frac{3 m_{\ell}^{2}}{8 q^{2}}\left(m_{D}^{2}-m_{P}^{2}\right)^{2}\left|f_{0}\left(q^{2}\right)\right|^{2}\right],
\end{aligned}
$$

where $E_{P}$ is the energy of the outgoing meson, $q$ is the total four-momentum transferred to the lepton pair, and the vector and scalar form factors $f_{+, 0}$, normalised such that $f_{+}(0)=f_{0}(0)$, parametrise the hadronic matrix element of the relevant charged current,

$$
\left\langle P\left|\bar{q} \gamma^{\mu} c\right| D\right\rangle=f_{+}\left(q^{2}\right)\left[p_{D}^{\mu}+p_{P}^{\mu}-\frac{m_{D}^{2}-m_{P}^{2}}{q^{2}} q^{\mu}\right]+f_{0}\left(q^{2}\right) \frac{m_{D}^{2}-m_{P}^{2}}{q^{2}} q^{\mu} .
$$



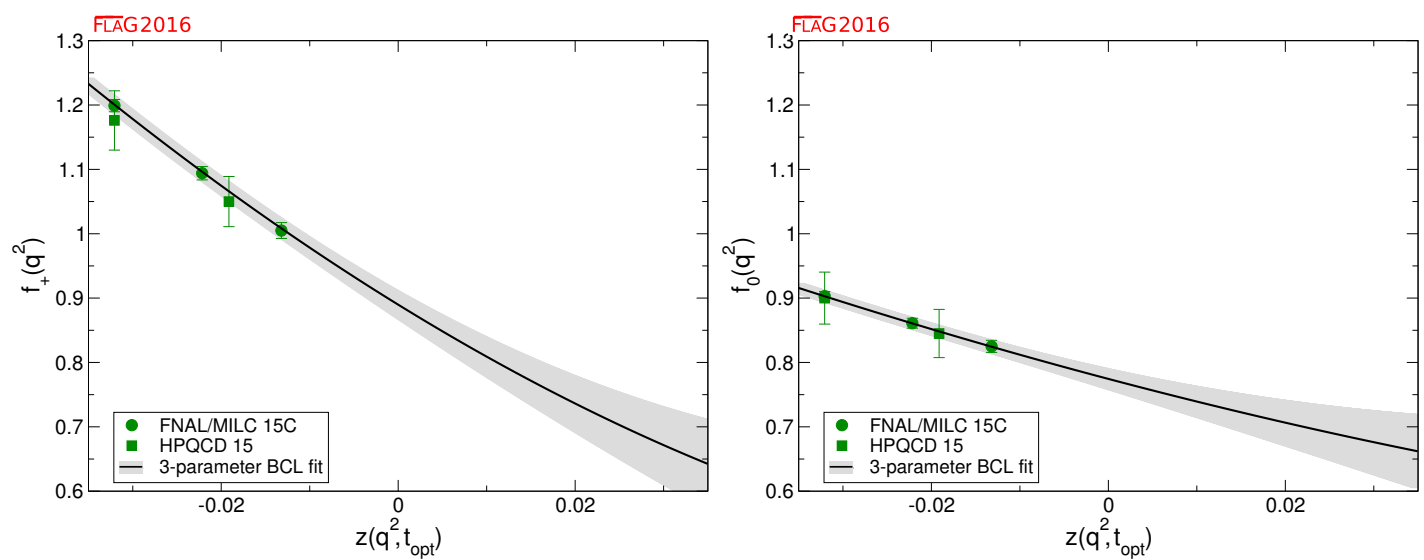

Figure 6: FLAG-3 BCL fits (grey band) for form factors $f_{+}\left(q^{2}\right)$ (left) and $f_{0}\left(q^{2}\right)$ (right) for $B \rightarrow D \ell v$, plotted versus $z\left(q^{2}\right)$. The $w=1$ point corresponds to $z \simeq-0.0323$. (See [3] for a complete list of references and a discussion of dataset and fit details.)

The contribution to Eq. (5.1) coming from $f_{0}$ is in practice negligible for $\ell=e, \mu$. Since, on the other hand, there are no experimental results for $\tau$ channels either in $D^{+}$or $D^{0}$ decay, ${ }^{5}$ lattice collaborations have focused on computations of the vector form factor $f_{+}$, which in turn allows to extract the value of the CKM matrix elements $\left|V_{c d}\right|$ and $\left|V_{c s}\right|$. While the kinematically allowed interval of values for $q^{2}$ is quite broad, all published lattice computations only provide the form factor at zero momentum transfer $f_{+}(0)$, which is sufficient to extract the CKM by comparing the form-factor normalisation or matching the total branching fraction for the process. Until recently, the PDG averages for the total branching fractions of these processes were dominated by the BaBar [47] and CLEO-c [48] measurements. New results from BESIII [29] will however result in a significant improvement. In particular, they provide very accurate determinations of the form-factor shapes (cf. Fig. 4 in [29]), that substantially improve on the CLEO-c, BaBar, and Belle existing results for the latter.

There is a marked paucity of lattice results for the form factors: both in FLAG-2 and FLAG-3 the only publications contributing to averages are the HPQCD $N_{\mathrm{f}}=2+1$ works [49]. New work is however being intensely pursued by other collaborations, and progress has been presented at this conference: they include the high-precision $N_{\mathrm{f}}=2+1+1$ computations by ETM [7, 50] and FNAL/MILC [6], and preliminary $N_{\mathrm{f}}=2+1$ results from JLQCD [8]. As we will discuss later, these are very interesting channels, both on their own phenomenological right, and for the purpose of understanding the systematic uncertainties involved in the description of the $q^{2}$ dependence of form factors in semileptonic heavy meson decay.

\section{$5.2 B_{(s)} \rightarrow D_{(s)}^{(*)} \ell v$ decays}

The most relevant exclusive modes used to determine $\left|V_{c b}\right|$ are the CKM-favoured $B_{(s)}$ semileptonic decays with a $D_{(s)}$ or a $D_{(s)}^{*}$ meson in the final state. Their SM rates for $\ell=e, \mu$ are given by ${ }^{6}$

$$
\begin{aligned}
\frac{\mathrm{d} \Gamma\left(B \rightarrow D \ell v_{\ell}\right)}{\mathrm{d} w} & =\frac{G_{\mathrm{F}}^{2} m_{D}^{3}}{48 \pi^{3}}\left(m_{B}+m_{D}\right)^{2}\left(w^{2}-1\right)^{3 / 2}\left|\eta_{\mathrm{EW}}\right|^{2}\left|V_{c b}\right|^{2}|\mathscr{G}(w)|^{2}, \\
\frac{\mathrm{d} \Gamma\left(B \rightarrow D^{*} \ell v_{\ell}\right)}{\mathrm{d} w} & =\frac{G_{\mathrm{F}}^{2} m_{D^{*}}^{3}}{4 \pi^{3}}\left(m_{B}-m_{D^{*}}\right)^{2}\left(w^{2}-1\right)^{1 / 2}\left|\eta_{\mathrm{EW}}\right|^{2}\left|V_{c b}\right|^{2} \chi(w)|\mathscr{F}(w)|^{2},
\end{aligned}
$$

\footnotetext{
${ }^{5}$ It is worth mentioning that, in the case of the $D_{s}$ meson, the only measured semileptonic mode with a pseudoscalar meson in the final state is $D_{s} \rightarrow K^{0} e^{+} v_{e}$, and the precision of the measurement is much poorer than in the case of the $D^{+, 0}$ semileptonic modes. These decays have therefore received little attention.

${ }^{6}$ In the following we drop $(s)$ subscripts; the channel being discussed will be clear by context, or explicitly indicated.
} 
where $w=p_{B} \cdot p_{D^{(*)}} /\left|p_{B} \cdot p_{D^{(*)}}\right|$ is the recoil parameter, $\eta_{\mathrm{EW}}$ contains electroweak loop corrections, and $\chi$ is a function of $w$ and meson masses that fulfills $\chi(1)=1$. In the case of the rate for $B \rightarrow$ $D \ell v$, Eq. (5.1) can be alternatively used with appropriate replacements in mass and CKM factors; the form factor $\mathscr{G}$ appearing in Eq. (5.3) is related to the standard vector form factor (cf. Eq. (5.2)) by $f_{+}\left(q^{2}\right)=\frac{1}{4}\left(1+\frac{m_{B}}{m_{D}}\right) \mathscr{G}(w)$, with $q^{2}=m_{B}^{2}+m_{D}^{2}-2 w m_{B} m_{D}$. Also of interest are the ratios of the total branching fractions in the $\tau$ channel relative to those in the light lepton channels, where CKM factors and electroweak corrections cancel, and which are strongly sensitive to the scalar form factors,

$$
R\left(D^{(*)}\right)=\frac{\mathscr{B}\left(B \rightarrow D^{(*)} \tau \nu_{\tau}\right)}{\mathscr{B}\left(B \rightarrow D^{(*)} \ell v_{\ell}\right)}
$$

Evidently, from the theory point of view the $D^{*}$ channel poses greater technical complications than the $D$ : the $D^{*}$ is unstable, which in principle requires a non-trivial procedure to extract the physical amplitude from an Euclidean correlation function; ${ }^{7}$ and a more complicated kinematics is involved.

Experimental measurements are, on the other hand, significantly more precise in the $D^{*}$ channel. ${ }^{8}$ The precision in $B \rightarrow D \ell v$ has however been significantly improved by a recent update by Belle [52], which is now the most precise measurement available. In the case of $\tau$ channels and the resulting values for $R\left(D^{(*)}\right)$, the experimental precision is again significantly better in the $D^{*}$ channel $(9.0 \% \mathrm{vs}$. $16.5 \%$ quoted in [53]), which has been further reinforced by a recent update by Belle [54] and a new measurement by $\mathrm{LHCb}$ [55]. The Belle II projection is to improve the precision on both observables by a factor of 4 to 5 with the full $50 \mathrm{ab}^{-1}$ dataset [32]. In the case of the channels with light leptons, the error is expected to be halved.

Lattice results with dynamical fermions had focused until recently on the form factors at zero recoil, and FLAG-2 reported $N_{\mathrm{f}}=2+1$ averages for $\mathscr{G}^{B \rightarrow D}(1)$ and $\mathscr{F}^{B \rightarrow D^{*}}(1)$, based on FNAL/MILC results [56]; $N_{\mathrm{f}}=2$ results for $\mathscr{G}^{B \rightarrow D}(1)$ and $\mathscr{G}^{B_{s} \rightarrow D_{s}}$ (1) obtained in [57] have also been published since then, and enter averages in FLAG-3. Recently, however, HPQCD [58] and FNAL/MILC [59] published first detailed $N_{\mathrm{f}}=2+1$ studies of form-factor shapes for both $f_{+}$and $f_{0}$, which in the latter case supersedes previous determinations of $\mathscr{G}^{B \rightarrow D}(1)$. Meanwhile, FNAL/MILC has also updated their $\mathscr{F}^{B \rightarrow D^{*}}(1)$ value [60]. This has led to a marked increase in the control over systematic uncertainties, resulting in more accurate exclusive determinations of $\left|V_{c b}\right|$ (see Section 6). The form factor determinations by HPQCD and FNAL/MILC can be averaged into a single function of $q^{2}$ (or $w$ ), using e.g. the FLAG-recommended BCL fit ansatz for the momentum transfer dependence (see Section 7); the result is illustrated in Fig. 6. Remarkably, as noted in [59], the $B \rightarrow D$ vector form-factor shape obtained for $N_{\mathrm{f}}=2+1$ is well-compatible with the quenched result from [61].

The availability of new results for the scalar form factor have also allowed for much more precise SM predictions for $R(D)$, with respect to the pre-existing FNAL/MILC value [62]; the resulting FLAG3 average is

$$
R(D)=0.300(8)
$$

It is worth stressing that no lattice-based computation of $R\left(D^{*}\right)$ is currently available. The commonly quoted SM prediction for $R\left(D^{*}\right)$ [63] is based on a phenomenological analysis that takes as input the experimental values for the form factors at zero recoil and the $w$ dependence in the heavy-quark limit, and estimates hadronic uncertainties using higher-order perturbative and power corrections to the latter. The resulting uncertainties largely cancel in $R\left(D^{*}\right)$, leading to an error much smaller than the one quoted for lattice determinations of $R(D)$.

\footnotetext{
${ }^{7}$ Note that this is very sensitive to light quark masses, since the $D^{*}$ mass is very close to the $D \pi$ threshold. In physical kinematics the width is in any case small enough so that its effect can likely be neglected at the current level of precision.

${ }^{8}$ The precision on the world average for the $B^{0} \rightarrow D^{-} \ell v$ total branching fraction quoted by the latest HFAG report is $4.5 \%$, while for $B^{0} \rightarrow D^{*-} \ell v$ is $2.2 \%$ - see [51] for a full discussion.
} 


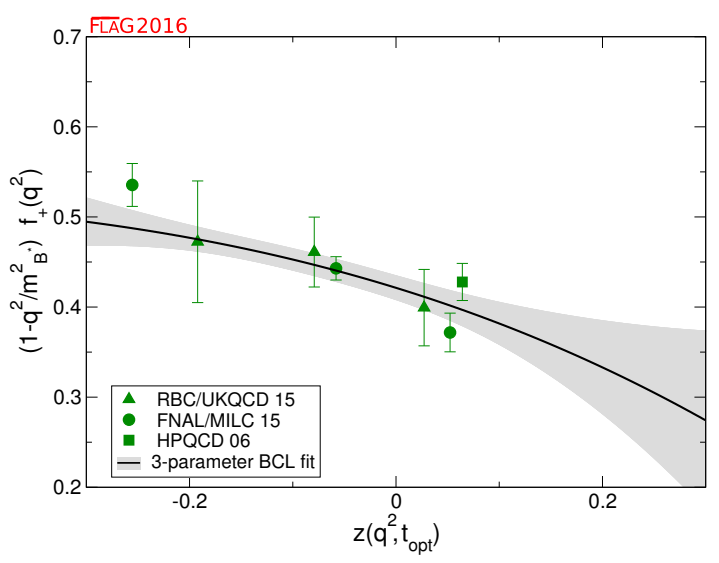

Figure 7: FLAG-3 BCL fit (grey band) for the form factor $f_{+}\left(q^{2}\right)$ for $B \rightarrow \pi \ell v$, plotted versus $z\left(q^{2}\right)$. (See [3] for a complete list of references and a discussion of dataset and fit details.)

\section{$5.3 B \rightarrow \pi \ell v$ and $B_{s} \rightarrow K \ell v$ decays}

The CKM-suppressed decay $B \rightarrow \pi \ell v_{\ell}$ is the most relevant exclusive channel for the determination of $\left|V_{u b}\right|$. Both it and the very similar $B_{s} \rightarrow K \ell v$ decay have SM differential rates given by Eq. (5.1), replacing $D \rightarrow B_{(s)}$ and $V_{c q} \rightarrow V_{u b}$; the relevant charged flavour current is $\bar{u} \gamma_{\mu} b$, which can again be parametrised by vector and scalar form factors as in Eq. (5.2).

The process $B \rightarrow \pi \ell v_{\ell}$ for light final leptons is well-controlled experimentally; the state-of-theart experimental dataset comprises various BaBar and Belle measurements, both untagged and with different tagging methods [64]. The HFAG average for the total branching fraction has a 3\% error [51], and the measured form-factor shapes show good agreement. Belle II projections foresee a precision improvement by a factor of 4 with the full $50 \mathrm{ab}^{-1}$ dataset, measured in terms of the error on $\left|V_{u b}\right|$ [32]. Meanwhile, the process $B_{s} \rightarrow K \ell v_{\ell}$ has not been measured yet, though it is expected to be within reach of the upcoming generation of $B$-physics results.

Until recently, only two $N_{\mathrm{f}}=2+1$ results for $B \rightarrow \pi \ell v$ were available, by FNAL/MILC [65] (addressing $f_{+}$only) and HPQCD [66] (with both $f_{+}$and $f_{0}$ ). The last two years saw however remarkable progress: FNAL/MILC significantly improved their previous determination, and produced results also for $f_{0}$ [67]; while RBC/UKQCD added their own independent determination of both form factors [68]. The latter paper also addresses $B_{s} \rightarrow K \ell v$, which, together with a previous publication by HPQCD [69], brings a SM prediction for the rate of this process. It is interesting to note that there is good consistency between all the computations, save for the scalar form factor in $B \rightarrow \pi \ell v$ : in that case, the pre-existing HPQCD determination exhibits a discrepancy with the new FNAL/MILC result (which is in turn consistent with the however less-precise RBC/UKQCD determination) at more than three standard deviations.

Fig. 7 shows the FLAG-3 averaged vector form factor for $B \rightarrow \pi \ell v$, using a BCL ansatz for the $q^{2}$ dependence (see Section 7); this result, strongly dominated by the new FNAL/MILC determination, allows for a significant improvement in the exclusive determination of $\left|V_{u b}\right|$ (cf. Section 6). Due to the discrepancies for $f_{0}$ mentioned above, FLAG-3 has instead not provided an average for the latter. In the case of $B_{s} \rightarrow K \ell v$, the good consistency of the results by HPQCD and RBC/UKQCD has instead allowed to average both form factors, illustrated in Fig. 8. In this case the precision for the vector form factor is comparable between the two computations, while the RBC/UKQCD determination of $f_{0}$ is much more precise than HPQCD's.

\section{$5.4 \Lambda_{b} \rightarrow \Lambda_{c} \ell v$ and $\Lambda_{b} \rightarrow p \ell v$ decays}

A very interesting new development in LQCD computations for heavy quark physics is the study of semileptonic decays of the $\Lambda_{b}$ baryon, with first unquenched results provided in a work by Detmold, 

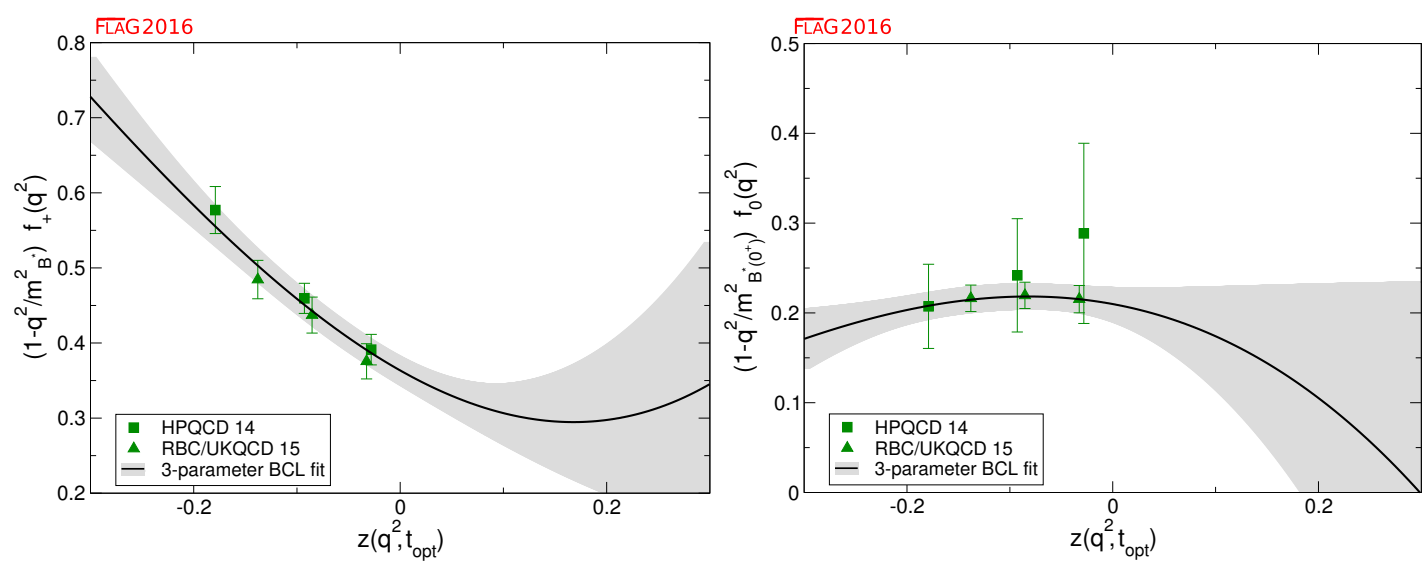

Figure 8: FLAG-3 BCL fits (grey band) for form factors $f_{+}\left(q^{2}\right)$ (left) and $f_{0}\left(q^{2}\right)$ (right) for $B_{s} \rightarrow K \ell v$, plotted versus $z\left(q^{2}\right)$. (See [3] for a complete list of references and a discussion of dataset and fit details.)

Lehner and Meinel [70]. The computation is based on RBC/UKQCD $N_{f}=2+1$ DWF ensembles, and treats the $b$ and $c$ quarks within the Columbia RHQ approach. The importance of this result is that, together with a recent analysis by $\mathrm{LHCb}$ of the ratio of decay rates $\Gamma\left(\Lambda_{b} \rightarrow p \ell v\right) / \Gamma\left(\Lambda_{b} \rightarrow \Lambda_{c} \ell v\right)$ [71], it allows for an exclusive determination of the ratio $\left|V_{u b}\right| /\left|V_{c b}\right|$ largely independent from the outcome of different exclusive channels, thus contributing a very interesting piece of information to the existing tensions in the determination of third-column CKM matrix elements (cf. Section 6).

The amplitudes of the decays $\Lambda_{b} \rightarrow p \ell v$ and $\Lambda_{b} \rightarrow \Lambda_{c} \ell v$ receive contributions from both the vector and the axial components of the current in the matrix elements $\left\langle p\left|\bar{q} \gamma^{\mu}\left(\mathbf{1}-\gamma_{5}\right) b\right| \Lambda_{b}\right\rangle$ and $\left\langle\Lambda_{c}\right| \bar{q} \gamma^{\mu}(\mathbf{1}-$ $\left.\gamma_{5}\right) b\left|\Lambda_{b}\right\rangle$, and can be parameterized in terms of six different form factors [72] — three in the parity-even sector and three in the parity-odd sector. All of them provide parametrically comparable contributions. Detmold et al. obtain results for all these form factors from suitable three-point functions, and fit them to a modified $z$-expansion ansatz (cf. Section 7) that combines the $q^{2}$ dependence with the chiral and continuum extrapolations. The relevant systematics is obviously very different with respect to the computations discussed above, since here baryonic channels are involved. The main results of the paper are the predictions for the individual form factor shapes and for the integrated rates in the interval of momentum transfer employed in the LHCb measurement. Prediction for the total rates in all possible lepton channels, as well as for ratios similar to $R(D)$ (cf. above) between the $\tau$ and light lepton channels are also available.

\subsection{Rare decays}

LQCD input is available for some exclusive semileptonic decay channels involving neutral-current $b \rightarrow$ $q$ transitions at the quark level, where $q=d, s$. Being forbidden at tree level in the SM, these processes allow for stringent tests of new physics; relevant examples are $B \rightarrow K^{*} \gamma, B \rightarrow K^{(*)} \ell^{+} \ell^{-}$, or $B \rightarrow \pi \ell^{+} \ell^{-}$.

The corresponding SM effective weak Hamiltonian is considerably more complicated than the one for tree-level processes: after neglecting top quark effects, as many as ten dimension-six operators formed by the product of two hadronic currents or one hadronic and one leptonic current appear. ${ }^{9}$ Three of the latter, coming from penguin and box diagrams, dominate at short distances; supplementing this with a combination of high-energy OPE arguments and results from Soft Collinear Effective Theory at intermediate energies, it is possible to argue that their contributions are still dominant when long-distance physics is also taken into account. Within this approximation, the dominant long-distance contribution thus consists of matrix elements of current operators (vector, tensor, and axial-vector) between one-hadron states, which in turn can be parameterized in terms of a number of form factors [74].

\footnotetext{
${ }^{9}$ See, e.g., [73] and references therein.
} 
On top of the aforementioned approximations, the lattice computation of the relevant form factors in channels with a vector meson in the final state faces extra challenges on top of those already present in the case of a pseudoscalar meson: the state is unstable, and the extraction of the relevant matrix element from correlation functions is significantly more complicated; and $\chi \mathrm{PT}$ cannot be used as a guide to extrapolate results at unphysically heavy pion masses to the chiral limit. While the field theory procedures to take resonance effects into account are available [75], they have not yet been implemented in the existing preliminary computations, which therefore suffer from an essentially uncontrolled source of systematic uncertainty. ${ }^{10}$

In decays to pseudoscalar mesons, there are results for the vector, scalar, and tensor form factors for $B_{s} \rightarrow K \ell^{+} \ell^{-}$decays by HPQCD [76] and FNAL/MILC [77], the latter paper also providing results for $B \rightarrow \pi \ell^{+} \ell^{-}$. Concerning channels with vector mesons in the final state, Horgan et al. have obtained the seven form factors relevant for $B \rightarrow K^{*} \ell^{+} \ell^{-}$(as well as those for $B_{s} \rightarrow \phi \ell^{+} \ell^{-}$) in [78] using NRQCD $b$ quarks and asqtad staggered light quarks. Finally, ongoing work on $B \rightarrow K^{*} \ell^{+} \ell^{-}$and $B_{s} \rightarrow \phi \ell^{+} \ell^{-}$by $\mathrm{RBC} / \mathrm{UKQCD}$, including first results, has recently been reported in [79].

\section{CKM matrix elements}

The LQCD-determined decay constants and form factors discussed above can be combined with the relevant experimental results to obtain exclusive values for the second- and third-row CKM matrix elements $\left|V_{c d}\right|,\left|V_{c s}\right|,\left|V_{u b}\right|$, and $\left|V_{c b}\right|$. In the case of $\left|V_{c q}\right|$, determinations are possible with results for $N_{\mathrm{f}}=2,2+1,2+1+1$; they mostly come from leptonic decays, with just one $N_{\mathrm{f}}=2+1$ semileptonic determination based on HPQCD's form factors. For $\left|V_{c b}\right|$ only semileptonic determinations based on $N_{\mathrm{f}}=2+1$ (two channels) and $N_{\mathrm{f}}=2$ (one channel) computations are possible. Finally, for $\left|V_{u b}\right|$ both semileptonic $\left(N_{\mathrm{f}}=2+1\right)$ and leptonic $\left(N_{\mathrm{f}}=2,2+1,2+1+1\right)$ determinations are possible; however, the latter come from the still poorly understood $B \rightarrow \tau \nu_{\tau}$ measurements, and have much larger errors than the semileptonic determination from $B \rightarrow \pi \ell v$. In the remainder of this section we will briefly summarise the FLAG-3 updated determination of these CKM matrix elements, based on the averages discussed in previous sections.

In order to determine $\left|V_{c d}\right|$ and $\left|V_{c s}\right|$, FLAG-3 combine their averages with PDG averages for $f_{D}\left|V_{c d}\right|$ and $f_{D_{s}}\left|V_{c s}\right|$ [31], and HFAG averages for $f_{+}^{D \rightarrow \pi}(0)\left|V_{c d}\right|$ and $f_{+}^{D \rightarrow K}(0)\left|V_{c s}\right|$ [51]. The result for all computations contributing to averages is illustrated in Fig. 9; the resulting average values are provided in Table 3. There is good consistency among all the determinations - in particular, no $N_{\mathrm{f}}$ dependence is apparent. The averages are consistent with $\left|V_{c d}\right|^{2}+\left|V_{c s}\right|^{2}+\left|V_{c b}\right|^{2}=1$ within at most two standard deviations; the level of precision makes this result independent of the value employed for $\left|V_{c b}\right|$.

In the case of $\left|V_{u b}\right|$ and $\left|V_{c b}\right|$, accurate determinations based on $B \rightarrow \pi \ell v$ and $B \rightarrow D \ell v$, respectively, can be obtained from simultaneous fits to the lattice vector form factors and state-of-the-art experimental data as a funcion of $q^{2}$, using a BCL ansatz (see Section 7) in which the CKM is left as a fitted relative normalisation. The outcome of this exercise, using as input the FLAG-3 averages for the form factors, is shown in Fig. 10. The resulting CKM values are shown in Fig. 11, together with leptonic determinations of $\left|V_{u b}\right|$ and the $\left|V_{c b}\right|$ determinations based on $B \rightarrow D^{*} \ell v$, as well as with inclusive determinations. The well-known tension between inclusive and exclusive values is still present with the latest generation of lattice results. FLAG-3 has decided not to quote a value for $\left|V_{u b}\right| /\left|V_{c b}\right|$ based on $\Lambda_{b}$ decays, since the lattice results for the latter do not meet some of the FLAG-3 requirements to enter averages or estimates. A summary of FLAG-3 quoted CKM values is provided in Table 4.

\section{The percent precision target}

As shown above, the precision level already attained for several observables of interest in heavy flavour physics on the lattice is at the few percent level. Exclusive CKM determinations bear errors generally

\footnotetext{
${ }^{10}$ This is a non-negligible effect e.g. in $B \rightarrow K^{*}$ transitions, given the $K^{*}$ width.
} 


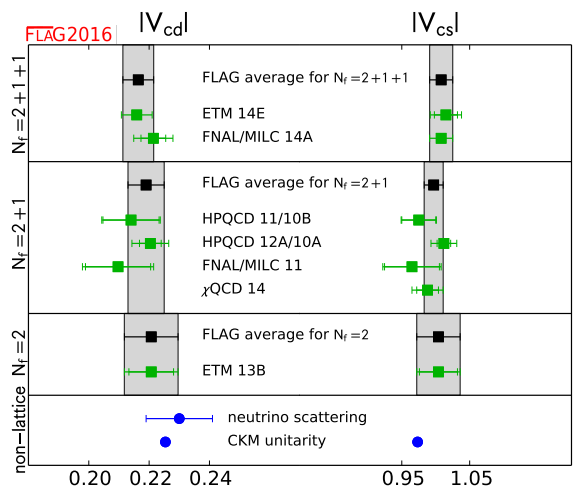

Figure 9: Determinations of $\left|V_{c d}\right|$ and $\left|V_{c s}\right|$ from values of charm decay constants and form factors entering FLAG-3 averages. (See [3] for a complete list of references.)

\begin{tabular}{lccc}
\hline \multicolumn{1}{c}{$N_{\mathrm{f}}$} & from & $\left|V_{c d}\right|$ & $\left|V_{c s}\right|$ \\
\hline $2+1+1$ & $f_{D}$ and $f_{D_{s}}$ & $0.2164(51)$ & $1.008(17)$ \\
$2+1$ & $f_{D}$ and $f_{D_{s}}$ & $0.2195(61)$ & $1.004(18)$ \\
2 & $f_{D}$ and $f_{D_{s}}$ & $0.2207(89)$ & $1.004(32)$ \\
\hline $2+1$ & $D \rightarrow \pi \ell v$ and $D \rightarrow K \ell v$ & $0.2140(97)$ & $0.975(26)$ \\
\hline $2+1$ & L+SL average & $0.2190(60)$ & $0.997(14)$ \\
\hline
\end{tabular}

Table 3: FLAG-3 determinations of $\left|V_{c d}\right|$ and $\left|V_{c s}\right|$. "L+SL" refers to the (correlated) average between the leptonic and semileptonic determinations.

better than $4 \%$, and the theory and experimental uncertainties are generally comparable. The leap on experimental precision expected from the current and upcoming generations of experiments will thus pose a stiff challenge to LQCD computations, which are expected in many cases to reach precisions in the 1-2\% ballpark. This is indeed already commonplace in computations in the pion and kaon sector; therefore, it is crucial to focus on the specific systematic uncertainties appearing in heavy flavour computations. One common aspect with light hadron physics is the need to consistently incorporate electromagnetic corrections, which are now the subject of intense work - see, e.g., [80]; or higherorder OPE contributions, which are already being considered in kaon observables [81]. Issues related to the difficulty to treat the $b$ quark within practical lattice regularisations, the correct treatment of resonances, or the use of perturbation theory, have already been briefly touched upon above.

The computation of semileptonic decay amplitudes has its own share of specific issues to be dealt with, like the effect of contributions from excited states [82], or the lack of an adequate chiral perturbation theory description in channels where final-state pseudoscalar mesons can have energies much larger than the chiral cutoff (e.g. $B \rightarrow \pi \ell v) .{ }^{11}$ One further key source of systematic uncertainties, which is now becoming crucial for several decay channels, is the description of the momentum transfer dependence of form factors; let us now conclude with a brief survey of this issue.

The benchmark channel where this systematics has long been studied is $B \rightarrow \pi \ell v$, since in this channel the kinematically allowed region in $q^{2}$ is broad enough so as to make a description in terms of a form factor at fixed $q^{2}$ impractical. Ansätze for the $q^{2}$ dependence are based on the generic observation that all form factors are analytic functions on the complex $q^{2}$ plane outside physical poles and inelastic threshold branch points; and this process is particularly simple, since the only resonance pole below the $B \pi$ production region is the (narrow) $B^{*}$, which is furthermore close to the threshold. Simple ansatz choices can thus be constructed using the idea of pole dominance; in particular, the Bećirević-

\footnotetext{
${ }^{11}$ See e.g. the discussion in [67], and the related works [83].
} 

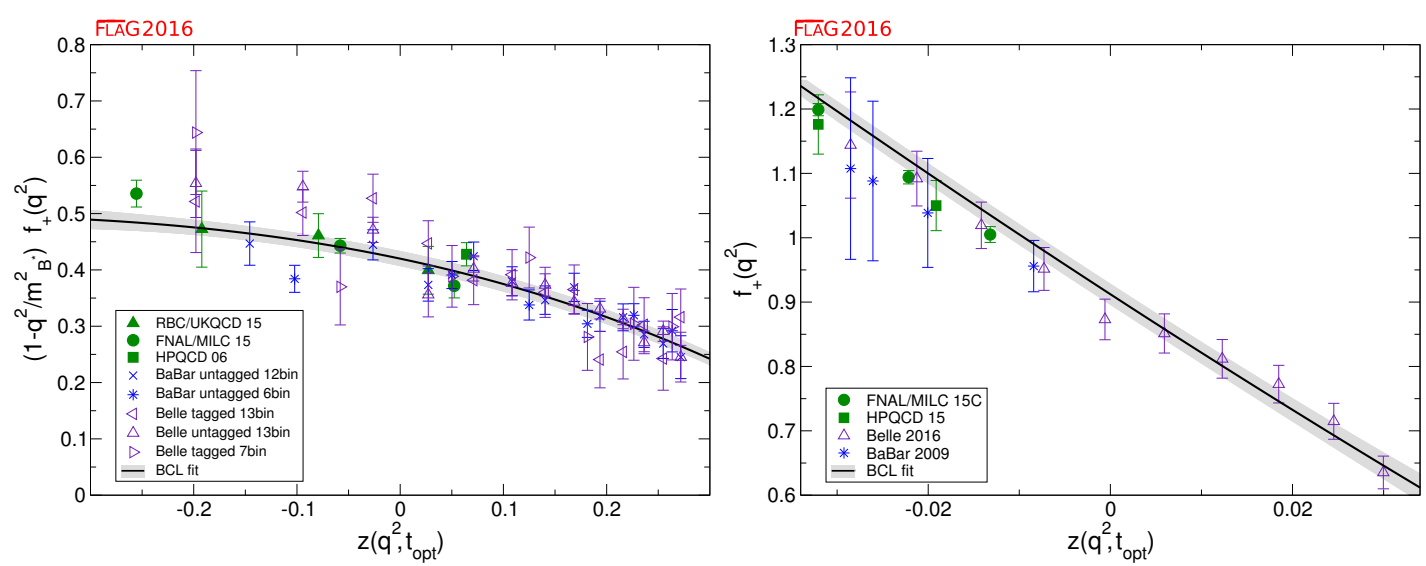

Figure 10: FLAG-3 BCL joint fits (grey band) to lattice and experimental form-factor data for $B \rightarrow \pi \ell v$ (left) and $B \rightarrow D \ell v$ (right), plotted versus $z\left(q^{2}\right)$. (See [3] for a complete list of references and a discussion of dataset and fit details.)

Kaidalov [84] and Ball-Zwicky [85] descriptions have been widely used. A more systematic approach exploits the positivity and analyticity properties of two-point functions of the vector current to obtain optimal parametrisations of form factors [86]. The general form of these so-called $z$-parametrisations is

$$
f\left(q^{2}\right)=\frac{1}{B\left(q^{2}\right) \phi\left(q^{2}, t_{0}\right)} \sum_{n=0}^{\infty} a_{n}\left(t_{0}\right) z\left(q^{2}, t_{0}\right)^{n}, \quad z\left(q^{2}, t_{0}\right)=\frac{\sqrt{t_{+}-q^{2}}-\sqrt{t_{+}-t_{0}}}{\sqrt{t_{+}-q^{2}}+\sqrt{t_{+}-t_{0}}},
$$

where the latter kinematical variable amounts to a conformal transformation of the complex $q^{2}$ plane, dependent on an essentially arbitrary parameter $t_{0}$, that maps the semileptonic region into a disc; $B\left(q^{2}\right)$ is a Blaschke factor that contains sub-threshold poles; and the outer function $\phi$ is some smooth positive function of $q^{2}$. The crucial property of this series expansion is that the coefficients $a_{n}$ satisfy the unitarity bound

$$
\sum_{n=0}^{\infty} a_{n}^{2}=\frac{1}{2 \pi i} \oint \frac{d z}{z}|B(z) \phi(z) f(z)|^{2}
$$

(where $\oint$ is taken over the image disc boundary in the $z$ plane). With a judicious choice for $\phi$ and $t_{0}$, this translates into a strong constraint, that allows to describe the form factor in terms of few free parameters, and a solid bound on the systematic uncertainty due to the series truncation. The simplest choice for the $B \rightarrow \pi$ vector form factor, dubbed BCL after the authors of [87], is

$$
f_{+}\left(q^{2}\right)=\frac{1}{1-q^{2} / m_{B^{*}}^{2}} \sum_{n=0}^{N} a_{n}\left(t_{0}\right) z\left(q^{2}, t_{0}\right)^{n}, \quad t_{0}=\left(m_{B}+m_{\pi}\right)\left(\sqrt{m_{B}}-\sqrt{m_{\pi}}\right)^{2},
$$

with the additional constraint $a_{N}=-\frac{(-1)^{N}}{N} \sum_{n=0}^{N-1}(-1)^{n} n a_{n}$ on the highest-order coefficient of the truncated series, that imposes the correct asymptotic behaviour at threshold. Eq. (7.3) is the FLAGrecommended parametrisation of choice for form factors.

The discussion above largely extends to the scalar form factor in $B \rightarrow \pi \ell v$ decays, as well as to form factors for other semileptonic transitions; a general discussion can be found, e.g., in [88]. The form factors for a generic $H \rightarrow L$ transition will display a cut starting at the production threshold $t_{+}$, and the optimal value of $t_{0}$ required in $z$-parameterizations is $t_{0}=t_{+}\left(1-\sqrt{1-t_{-} / t_{+}}\right)$(where $t_{ \pm}=$ $\left.\left(m_{H} \pm m_{L}\right)^{2}\right)$. For unitarity bounds to apply, the Blaschke factor has to include all sub-threshold poles with the quantum numbers of the hadronic current - e.g., vector (resp. scalar) resonances with $b \bar{u}$ quark content for the $B \rightarrow \pi, B_{s} \rightarrow K$ vector (resp. scalar) form factors; and idem $b \bar{c}$ quark content for 

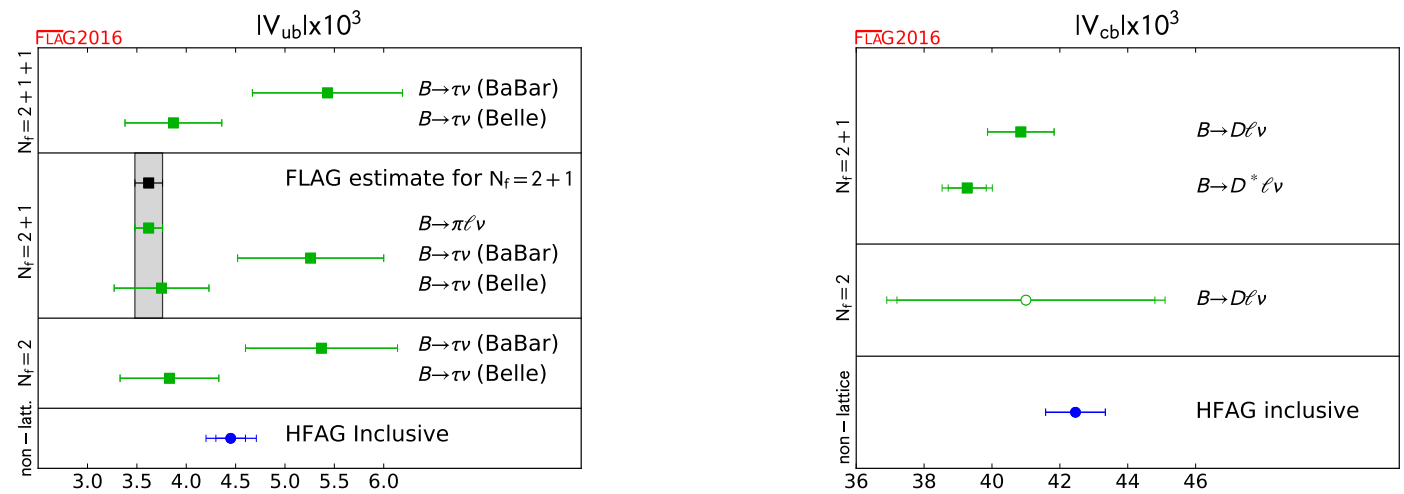

Figure 11: FLAG-3 summary plots for the determination of $\left|V_{u b}\right|$ and $\left|V_{c b}\right|$ (see [3] for a complete list of references).

\begin{tabular}{l|cc|cc}
\hline \multicolumn{1}{c|}{$N_{\mathrm{f}}$} & from & $\left|V_{u b}\right|$ & from & $\left|V_{c b}\right|$ \\
\hline \multirow{2}{*}{$2+1$} & $B \rightarrow \pi \ell v$ & $3.62(14) \times 10^{-3}$ & $B \rightarrow D \ell v$ & $40.85(98) \times 10^{-3}$ \\
& & & $B \rightarrow D^{*} \ell v$ & $39.27(74) \times 10^{-3}$ \\
\hline 2 & & & $B \rightarrow D \ell v$ & $41.0(4.1) \times 10^{-3}$ \\
\hline
\end{tabular}

Table 4: FLAG-3 determinations of $\left|V_{u b}\right|$ and $\left|V_{c b}\right|$.

$B \rightarrow D .{ }^{12}$ Thus, as emphasized above, the control over systematic uncertainties brought in by using $z$-parametrisations strongly depends on implementation details. This has practical consequences, in particular, when the resonance spectrum in a given channel is not sufficiently well-known, or resonances are very close to thresholds, and may go across them as quark masses are changed. Caveats may also apply for channels where resonances with a non-negligible width appear. A further issue is whether $t_{+}=\left(m_{H}+m_{L}\right)^{2}$ is the proper choice for the start of the cut in cases such as $B_{s} \rightarrow K \ell v$ and $B \rightarrow D \ell v$, where there are lighter two-particle states that project on the current ( $B \pi$ and $B_{c} \pi$, respectively). In any such situation, it is not clear a priori that a given $z$-parametrisation will satisfy strict bounds, as has been seen, e.g., in determinations of the proton charge radius from electron-proton scattering [89]. One particular case where several of these issues may be at play regards the use of so-called modified $z$-expansions, pioneered by HPQCD, that take into account simultaneously the $q^{2}$, light quark masses, and lattice spacing dependence of form factors. Because the modified $z$-expansion is not derived from an underlying effective field theory, it is unclear how systematic uncertainties can be quantified within this approach - in particular, the applicability of unitarity bounds has to be examined carefully.

The rapid growth in the number of lattice and experimental results for semileptonic decays where an accurate description of the $q^{2}$ dependence is needed warrants an in-depth general study of how these ideas can be optimally applied to as many channels as possible. One particularly interesting case, from the methodological point of view, is the recent extraction of accurate $D \rightarrow \pi$ and $D \rightarrow K$ form factors by BESIII [29], where the dominant source of experimental uncertainty in view of CKM determinations comes from the description of the $q^{2}$ dependence. Since the relevant vector resonances are extremely close to the inelastic threshold, these channels constitute an excellent laboratory to deepen into a number of the issues pointed out above.

\section{Conclusions and outlook}

There is rapid progress in LQCD relevant for heavy quark physics - especially so, during the last two years, in addressing semileptonic $b$ decays. For several observables of interest (e.g. those involved in the

\footnotetext{
${ }^{12} \mathrm{~A}$ more complicated analytic structure may arise in other cases, such as channels with vector mesons in the final state.
} 
determination of CKM matrix elements) the theory uncertainty is now comparable to, or smaller than, the experimental one. There is also a fertile interaction between lattice and experimental collaborations, which allows to better focus efforts on both sides; the recent study of $\Lambda_{b}$ decays is an excellent success story in this respect.

The upcoming era of experimental results, led by LHCb, BESIII and, especially, Belle II, will however pose a strong challenge to the accuracy of lattice methods. In particular, the systematic uncertainties related to the treatment of $b$ quarks will take a central role, and it will become crucial to have as many cross-checks as possible among different procedures. Also, many small effects which have been up to now neglected or not fully addressed - from electromagnetic corrections to various field-theory aspects (use of perturbation theory, chiral extrapolations, $q^{2}$ dependence of form factors, resonance effects) - will become relevant. Incorporating state-of-the-art ensembles already used for pion and kaon physics to new heavy-flavour studies will play a role in decreasing various uncertainties, too.

Ultimately, however, it is of the utmost importance to diminish our reliance on effective theories by being able to directly simulate physical $b$ and light quarks simultaneously. Important steps in that direction are being taken $[12,14,15]$, and should have a key role in planning for new $N_{\mathrm{f}}=2+1+1(+1)$ simulations in the near future.

\section{Acknowledgements}

Support from the EU PITN-GA-2009-238353 (STRONGnet), MCINN grants FPA2012-31686 and FPA2012-31880, and MINECO's "Centro de Excelencia Severo Ochoa" Programme under grant SEV2012-0249, is gratefully acknowledged. In the preparation of my talk I benefited from discussions with, and input from, C.T.H. Davies, C. DeTar, P. Dimopoulos, D. Du, G. Herdoíza, T. Ishikawa, A. Kronfeld, P. Lami, K. Nakayama, R. Sommer, J.T. Tsang, C. Urbach, R. Van de Water, and Y. Yang. I am indebted to my FLAG colleagues for the huge amount of work put in producing the review, and for many illuminating discussions. A critical reading of a first version of this writeup by P. Fritzsch and G. Herdoíza has greatly contributed towards improving it. Finally, I would like to thank the Lattice 2015 organisers for a splendid conference, in the face of adverse elements. お疲れ様でした。

\section{References}

[1] T. Iijima, this conference.

[2] G. Colangelo et al., Eur. Phys. J. C 71 (2011) 1695; S. Aoki et al., ibid. 74 (2014) 2890.

[3] S. Aoki et al., arXiv:1607.00299 [hep-lat].

[4] S. Meinel, this conference; P. Korcyl, C. Lehner and T. Ishikawa, this conference, PoS LATTICE 2015 (2015) 254; C. Wittemeier, this conference; F. Knechtli et al., this conference, arXiv:1511.04914 [hep-lat]; A.T. Lytle [LATTICE-HPQCD Collaboration], this conference, arXiv:1511.06547 [hep-lat]; K. Nakayama, B. Fahy and S. Hashimoto, this conference, arXiv:1511.09163 [hep-lat].; T. Ishikawa, this conference; J. Simone, this conference; R. Zhou, this conference; B. Colquhoun et al., this conference, arXiv:1511.01852 [hep-lat]; P. Boyle et al., this conference, arXiv:1511.09328 [hep-lat]; T. Kawanai, this conference; J. Flynn et al., this conference, arXiv:1511.06622 [hep-lat]; O. Witzel, this conference;

A. Gérardin, this conference; J. Leem, this conference; M. Wurtz, R. Lewis and R.M. Woloshyn, this conference, arXiv: 1510.05445 [hep-lat]; Y.C. Koch, this conference.

[5] A. Bazavov et al. [MILC and Fermilab Lattice Collaborations], this conference, arXiv:1511.02294 [hep-lat].

[6] T. Primer et al. [MILC and Fermilab Lattice Collaborations], this conference, arXiv:1511.04000 [hep-lat].

[7] N. Carrasco et al., this conference, arXiv:1511.04877 [hep-lat].

[8] T. Suzuki, this conference.

[9] L. Giusti, PoS LAT 2006 (2006) 009.

[10] L. Del Debbio, H. Panagopoulos and E. Vicari, JHEP 0208 (2002) 044; S. Schaefer et al. [ALPHA Collaboration], Nucl. Phys. B 845 (2011) 93.

[11] S. Gottlieb, this conference. 
[12] M. Lüscher and S. Schaefer, JHEP 1107 (2011) 036; Comput. Phys. Commun. 184 (2013) 519.

[13] M. Bruno et al., JHEP 1502 (2015) 043.

[14] S. Mages et al., arXiv:1512.06804 [hep-lat].

[15] M.G. Endres et al., Phys. Rev. D 92 (2015) 114516.

[16] N. Isgur and M.B. Wise, Phys. Lett. B 232 (1989) 113; E. Eichten and B.R. Hill, ibid. 234 (1990) 511; N. Isgur and M.B. Wise, ibid. 237 (1990) 527.

[17] W.E. Caswell and G.P. Lepage, Phys. Lett. B 167 (1986) 437; G.T. Bodwin, E. Braaten and G.P. Lepage, Phys. Rev. D 51 (1995) 1125 Erratum: [Phys. Rev. D 55 (1997) 5853].

[18] J. Heitger et al. [ALPHA Coll.], JHEP 0402 (2004) 022; B. Blossier et al., ibid. 1006 (2010) 002.

[19] R. Sommer, hep-lat/0611020; M. Della Morte, PoS LAT 2007 (2007) 008.

[20] B.A. Thacker and G.P. Lepage, Phys. Rev. D 43 (1991) 196; G.P. Lepage et al., ibid. 46 (1992) 4052.

[21] E.B. Gregory et al., Phys. Rev. D 83 (2011) 014506.

[22] A.X. El-Khadra, A.S. Kronfeld and P.B. Mackenzie, Phys. Rev. D 55 (1997) 3933.

[23] N.H. Christ, M. Li and H.W. Lin, Phys. Rev. D 76 (2007) 074505.

[24] S. Aoki, Y. Kuramashi and S.i. Tominaga, Prog. Theor. Phys. 109 (2003) 383.

[25] B. Sheikholeslami and R. Wohlert, Nucl. Phys. B 259 (1985) 572.

[26] G.M. de Divitiis et al., Nucl. Phys. B 675 (2003) 309; D. Guazzini, R. Sommer and N. Tantalo, PoS LAT 2006 (2006) 084; B. Blossier et al. [ETM Collaboration], PoS LAT 2009 (2009) 151.

[27] B. Blossier et al. [ETM Collaboration], JHEP 1004 (2010) 049.

[28] R. Sommer, arXiv:1008.0710 [hep-lat]; P. Fritzsch et al. [ALPHA Collaboration], JHEP 1601 (2016) 093.

[29] H. Ma [BESIII Collaboration], arXiv:1509.04424 [hep-ex].

[30] S. Eidelman, arXiv:1509.06838 [hep-ex].

[31] K. A. Olive et al. [Particle Data Group Collaboration], Chin. Phys. C 38 (2014) 090001.

[32] BELLE2-NOTE-PH-2015-002, retrieved from B2TiP.

[33] A. Bazavov et al. [Fermilab Lattice and MILC Collaborations], Phys. Rev. D 90 (2014) 074509.

[34] N. Carrasco et al. [ETM Collaboration], JHEP 1403 (2014) 016.

[35] Y. B. Yang et al., Phys. Rev. D 92 (2015) 034517.

[36] W. P. Chen et al. [TWQCD Collaboration], Phys. Lett. B 736 (2014) 231.

[37] V. Khachatryan et al. [CMS and LHCb Collaborations], Nature 522 (2015) 68.

[38] B. Aubert et al. [BaBar Collaboration], Phys. Rev. D 81 (2010) 051101; J. P. Lees et al. [BaBar Collaboration], ibid. 88 (2013) 031102.

[39] I. Adachi et al. [Belle Collaboration], Phys. Rev. Lett. 110 (2013) 131801; B. Kronenbitter et al. [Belle Collaboration], Phys. Rev. D 92 (2015) 051102.

[40] C. McNeile et al., Phys. Rev. D 86 (2012) 074503.

[41] F. Bernardoni et al. [ALPHA Collaboration], Phys. Lett. B 735 (2014) 349.

[42] N.H. Christ et al., Phys. Rev. D 91 (2015) 054502.

[43] Y. Aoki et al., Phys. Rev. D 91 (2015) 114505.

[44] N. Carrasco et al., PoS LATTICE 2013 (2014) 313.

[45] E. Gámiz et al. [HPQCD Collaboration], Phys. Rev. D 80 (2009) 014503.

[46] C.M. Bouchard et al., PoS LATTICE 2011 (2011) 274.

[47] B. Aubert et al. [BaBar Collaboration], Phys. Rev. D 76 (2007) 052005; J.P. Lees et al. [BaBar Collaboration], ibid 91 (2015) 052022.

[48] D. Besson et al. [CLEO Collaboration], Phys. Rev. D 80 (2009) 032005.

[49] H. Na et al., Phys. Rev. D 82 (2010) 114506; H. Na et al., ibid 84 (2011) 114505.

[50] N. Carrasco el al., arXiv:1602.04113 [hep-lat].

[51] Y. Amhis et al. [Heavy Flavor Averaging Group (HFAG) Collaboration], arXiv:1412.7515 [hep-ex].

[52] R. Glattauer et al. [Belle Collaboration], Phys. Rev. D 93 (2016) 032006.

[53] J.P. Lees et al. [BaBar Collaboration], Phys. Rev. D 88 (2013) 072012.

[54] M. Huschle et al. [Belle Collaboration], Phys. Rev. D 92 (2015) 072014.

[55] R. Aaij et al. [LHCb Collaboration], Phys. Rev. Lett. 115 (2015) 111803 Addendum: [Phys. Rev. Lett. 115 (2015) 159901].

[56] M. Okamoto et al., Nucl. Phys. Proc. Suppl. 140 (2005) 461; C. Bernard et al., Phys. Rev. D 79 (2009) 014506. 
[57] M. Atoui et al., Eur. Phys. J. C 74 (2014) 2861.

[58] H. Na et al. [HPQCD Collaboration], Phys. Rev. D 92 (2015) 054510.

[59] J.A. Bailey et al. [MILC Collaboration], Phys. Rev. D 92 (2015) 034506.

[60] J.A. Bailey et al. [Fermilab Lattice and MILC Collaborations], Phys. Rev. D 89 (2014) 114504.

[61] G.M. de Divitiis et al., Phys. Lett. B 655 (2007) 45.

[62] J.A. Bailey et al., Phys. Rev. Lett. 109 (2012) 071802.

[63] S. Fajfer, J.F. Kamenik and I. Nisandzic, Phys. Rev. D 85 (2012) 094025.

[64] P. del Amo Sánchez et al. [BaBar Collaboration], Phys. Rev. D 83 (2011) 032007; J.P. Lees et al. [BaBar Collaboration], ibid 86 (2012) 092004; H. Ha et al. [Belle Collaboration], ibid 83 (2011) 071101;

A. Sibidanov et al. [Belle Collaboration], ibid 88 (2013) 032005.

[65] J.A. Bailey et al., Phys. Rev. D 79 (2009) 054507.

[66] E. Dalgic et al., Phys. Rev. D 73 (2006) 074502 Erratum: [Phys. Rev. D 75 (2007) 119906].

[67] J.A. Bailey et al. [Fermilab Lattice and MILC Collaborations], Phys. Rev. D 92 (2015) 014024.

[68] J.M. Flynn et al., Phys. Rev. D 91 (2015) 074510.

[69] C.M. Bouchard et al., Phys. Rev. D 90 (2014) 054506.

[70] W. Detmold, C. Lehner and S. Meinel, Phys. Rev. D 92 (2015) 034503.

[71] R. Aaij et al. [LHCb Collaboration], Nature Phys. 11 (2015) 743.

[72] T. Feldmann and M.W.Y. Yip, Phys. Rev. D 85 (2012) 014035 Erratum: [Phys. Rev. D 86 (2012) 079901$].$

[73] M. Antonelli et al., Phys. Rept. 494 (2010) 197.

[74] Z. Liu et al., PoS LAT 2009 (2009) 242.

[75] M. Lüscher, Commun. Math. Phys. 105 (1986) 153; M. Lüscher, Nucl. Phys. B 354 (1991) 531; M. Lüscher, Nucl. Phys. B 364 (1991) 237; M. Lage, U.G. Meissner and A. Rusetsky, Phys. Lett. B 681 (2009) 439; V. Bernard et al., JHEP 1101 (2011) 019; M. Doring et al., Eur. Phys. J. A 47 (2011) 139; M.T. Hansen and S.R. Sharpe, Phys. Rev. D 86 (2012) 016007; R.A. Briceño and Z. Davoudi, Phys. Rev. D 88 (2013) 094507; J.J. Dudek et al. [Hadron Spectrum Collaboration], Phys. Rev. Lett. 113 (2014) 182001.

[76] C. Bouchard et al. [HPQCD Collaboration], Phys. Rev. D 88 (2013) 054509 Erratum: [Phys. Rev. D 88 (2013) 079901].

[77] J.A. Bailey et al., Phys. Rev. D 93 (2016) 025026.

[78] R.R. Horgan et al., Phys. Rev. D 89 (2014) 094501.

[79] J. Flynn et al., arXiv:1511.06622 [hep-lat].

[80] A. Duncan, E. Eichten and H. Thacker, Phys. Rev. Lett. 76 (1996) 3894; G.M. de Divitiis et al. [RM123 Collaboration], Phys. Rev. D 87 (2013) 114505; S. Basak et al. [MILC Collaboration], PoS LATTICE 2014 (2014) 116; T. Ishikawa et al., Phys. Rev. Lett. 109 (2012) 072002; S. Aoki et al., Phys. Rev. D 86 (2012) 034507; T. Blum et al., Phys. Rev. D 82 (2010) 094508; N. Tantalo, PoS LATTICE 2013 (2014) 007; A. Portelli, PoS LATTICE 2014 (2015) 013; N. Carrasco et al., Phys. Rev. D 91 (2015) 074506; Z. Fodor et al., Phys. Lett. B 755 (2016) 245; B. Lucini et al., JHEP 1602 (2016) 076.

[81] Z. Bai et al., Phys. Rev. Lett. 113 (2014) 112003; N.H. Christ et al. [RBC and UKQCD Collaborations], Phys. Rev. D 92 (2015) 094512; N.H. Christ et al., arXiv:1605.04442 [hep-lat].

[82] F. Bahr et al. [ALPHA Collaboration], Phys. Lett. B 757 (2016) 473.

[83] J. Bijnens and I. Jemos, Nucl. Phys. B 840 (2010) 54 Erratum: [Nucl. Phys. B 844 (2011) 182]; J. Bijnens and I. Jemos, Nucl. Phys. B 846 (2011) 145; M. Procura et al., PoS CD 12 (2013) 049.

[84] D. Bećirević and A. B. Kaidalov, Phys. Lett. B 478 (2000) 417.

[85] P. Ball and R. Zwicky, Phys. Rev. D 71 (2005) 014015.

[86] C. Bourrely, B. Machet and E. de Rafael, Nucl. Phys. B 189 (1981) 157; C.G. Boyd, B. Grinstein and R.F. Lebed, Phys. Rev. Lett. 74 (1995) 4603; L. Lellouch, Nucl. Phys. B 479 (1996) 353; C.G. Boyd and M.J. Savage, Phys. Rev. D 56 (1997) 303; M.C. Arnesen et al., Phys. Rev. Lett. 95 (2005) 071802; T. Becher and R.J. Hill, Phys. Lett. B 633 (2006) 61.

[87] C. Bourrely, I. Caprini and L. Lellouch, Phys. Rev. D 79 (2009) 013008. Erratum: [Phys. Rev. D 82 (2010) 099902].

[88] R.J. Hill, eConf C 060409 (2006) 027.

[89] R.J. Hill and G. Paz, Phys. Rev. D 82 (2010) 113005; R.J. Hill and G. Paz, Phys. Rev. Lett. 107 (2011) 160402; Z. Epstein, G. Paz and J. Roy, Phys. Rev. D 90 (2014) 074027. 